\title{
Highly boosted Higgs bosons and unitarity in vector-boson fusion at future hadron colliders
}

\author{
Wolfgang Kilian, ${ }^{a}$ Sichun Sun, ${ }^{b, c}$ Qi-Shu Yan, ${ }^{d, e}$ Xiaoran Zhao ${ }^{f, g}$ and Zhijie Zhao ${ }^{e}$ \\ ${ }^{a}$ Department of Physics, University of Siegen, \\ Walter-Flex-Straße 3, Siegen 57068, Germany \\ ${ }^{b}$ Department of Physics and INFN, Sapienza University of Rome, \\ Piazzale Aldo Moro, 5, Roma 00185, Italy \\ ${ }^{c}$ School of Physics, Beijing Institute of Technology, \\ 5 Zhongguancun St, Haidian District, Beijing 100081, China \\ ${ }^{d}$ School of Physics, University of Chinese Academy of Sciences, \\ 19A Yuquan Road, Beijing 100039, China \\ ${ }^{e}$ Center for Future High Energy Physics, Institute of High Energy Physics, \\ Chinese Academy of Sciences, 19B Yuquan Road, Beijing 100039, China \\ ${ }^{f}$ Centre for Cosmology, Particle Physics and Phenomenology (CP3), \\ Université Catholique de Louvain, Chemin du Cyclotron, 2, Louvain-la-Neuve B-1348, Belgium \\ ${ }^{g}$ Dipartimento di Matematica e Fisica, Università di Roma Tre and \\ INFN, sezione di Roma Tre, \\ Via della Vasca Navale 84, Rome I-00146, Italy \\ E-mail: kilian@physik.uni-siegen.de, sichunssun@gmail.com, \\ yanqishu@ucas.ac.cn, xiaoran.zhao@uniroma3.it, \\ zhao@physik.uni-siegen.de
}

ABSTRACT: We study the observability of new interactions which modify Higgs-pair production via vector-boson fusion processes at the LHC and at future proton-proton colliders. In an effective-Lagrangian approach, we explore in particular the effect of the operator $h^{2} W_{\mu \nu}^{a} W^{a, \mu \nu}$, which describes the interaction of the Higgs boson with transverse vectorboson polarization modes. By tagging highly boosted Higgs bosons in the final state, we determine projected bounds for the coefficient of this operator at the LHC and at a future $27 \mathrm{TeV}$ or $100 \mathrm{TeV}$ collider. Taking into account unitarity constraints, we estimate the new-physics discovery potential of Higgs pair production in this channel.

Keywords: Effective Field Theories, Higgs Physics

ArXiv EPrint: 2101.12537 


\section{Contents}

1 Introduction 1

2 Higgs pair production in the Standard Model 3

2.1 Analysis method at the $14 \mathrm{TeV}$ LHC 4

2.1.1 The mass drop method for highly-boosted Higgs boson tagging 4

$\begin{array}{lll}2.1 .2 & \text { Multivariate analysis } & 7\end{array}$

2.2 Analysis of $p p \rightarrow h h j j$ in SM at $100 \mathrm{TeV}$ hadron collider 9

3 Multi-Higgs production in VBF processes with dimension-6 operators 10

$\begin{array}{lll}3.1 & \text { Effective-theory description } & 11\end{array}$

3.2 Higgs-pair couplings to transverse vector polarizations in VBF 13

$\begin{array}{ll}3.3 & \text { Unitarity limits and discovery reach } \\ \end{array}$

$\begin{array}{lll}4 & \text { Two-parameter bounds } & 17\end{array}$

5 Discussions and conclusions $\quad 22$

\section{Introduction}

In 2012, the Higgs boson was discovered at the LHC [1, 2]. Current experimental data show that its properties agree with the predictions of Standard Model (SM), but further measurements are still necessary to examine the SM and search for new physics.

The SM predicts that the Higgs boson has three kinds of interaction at tree level: (1) the Yukawa interaction with fermions; $(2)$ the interaction with massive vector bosons $\left(W^{ \pm}\right.$ and $Z) ;(3)$ the cubic and quartic Higgs self-interactions. One of the prime targets of the second run and future runs of the LHC is to measure the Higgs self-couplings (3).

Higgs pair-production in vector-boson fusion (VBF) [3], $V V \rightarrow h h$, is sensitive to the last two kinds of interaction. VBF-type processes are possible at both electron-positron and hadron colliders. At a hadron collider, the two incoming vector bosons $V=W^{ \pm}, Z$ are radiated from two initial quarks. In the final state, in addition to two Higgs bosons, there are two back-to-back hard jets which should be tagged in the forward and backward regions of a detector, respectively. This allows us to use VBF cuts to reject the QCD type backgrounds efficiently.

In this paper, we study double Higgs production in the VBF process in an effectivefield theory (EFT) approach. We follow the conventions given in our previous paper [4], 
and use the following phenomenological effective Lagrangian:

$$
\begin{aligned}
\mathcal{L}_{\mathrm{EFT}}= & \mathcal{L}_{\overline{\mathrm{SM}}}+\mathcal{L}_{V V h}+\mathcal{L}_{V h}, \\
\mathcal{L}_{V V h}= & -\left(g_{W, b 1} \frac{h}{v}+g_{W, b 2} \frac{h^{2}}{2 v^{2}}+g_{W, b 3} \frac{h^{3}}{6 v^{3}}+\cdots\right) W_{\mu \nu}^{+} W^{-\mu \nu} \\
& -\left(g_{A, b 1} \frac{h}{2 v}+g_{A, b 2} \frac{h^{2}}{4 v^{2}}+g_{A, b 3} \frac{h^{3}}{12 v^{3}}+\cdots\right) F_{\mu \nu} F^{\mu \nu} \\
& -\left(g_{X, b 1} \frac{h}{v}+g_{X, b 2} \frac{h^{2}}{2 v^{2}}+g_{X, b 3} \frac{h^{3}}{6 v^{3}}+\cdots\right) F_{\mu \nu} Z^{\mu \nu} \\
& -\left(g_{Z, b 1} \frac{h}{2 v}+g_{Z, b 2} \frac{h^{2}}{4 v^{2}}++g_{Z, b 3} \frac{h^{3}}{12 v^{2}}+\cdots\right) Z_{\mu \nu} Z^{\mu \nu} \\
\mathcal{L}_{V H}= & g_{W, a 1} \frac{2 m_{W}^{2}}{v} h W^{+, \mu} W_{\mu}^{-}+g_{W, a 2} \frac{m_{W}^{2}}{v^{2}} h^{2} W^{\mu} W_{\mu}+g_{W, a 3} \frac{m_{W}^{2}}{3 v^{3}} h^{3} W^{\mu} W_{\mu} \\
& +g_{Z, a 1} \frac{m_{Z}^{2}}{v} h Z^{\mu} Z_{\mu}+g_{Z, a 2} \frac{m_{Z}^{2}}{2 v^{2}} h^{2} Z^{\mu} Z_{\mu}+g_{Z, a 3} \frac{m_{Z}^{2}}{6 v^{3}} h^{3} Z^{\mu} Z_{\mu}+\cdots
\end{aligned}
$$

Dots indicate higher-dimensional interactions which are not relevant for the VBF Higgs production process that we consider. We only include the CP-conserving interactions and omit any CP-violating operators. The relations $g_{W, a 1}=g_{W, a 2}=g_{Z, a 1}=g_{Z, a 2}=1$ and $g_{V, b 1}=g_{V, b 2}=g_{V, b 3}=g_{W, a 3}=g_{Z, a 3}=0$ characterize the SM reference values at the tree level, where the subscript letter $V$ denotes $W, A, X, Z$, respectively. The corresponding terms have been removed from the SM Lagrangian, as indicated by the overline notation $\overline{\mathrm{SM}}$, such that they are not double-counted.

Introducing gauge degrees of freedom in this phenomenological Lagrangian, the vertices can be rewritten as gauge-invariant operators which are understood as the low-energy effect of short-range structure or new heavy degrees of freedom beyond the SM. Such effects would appear, for instance, in a composite Higgs model — for concrete examples, cf. [5-8]. In section 3, we relate the parameterization to the formulation in terms of gauge-invariant operators, adopting a concrete basis and truncating the expansion at dimension six, as commonly done in the literature.

The single Higgs couplings $g_{V, a 1}$ can be determined to up to $5-10 \%$ via measuring the decay fractions $h \rightarrow W W^{*}$ and $h \rightarrow Z Z^{*}$ at the LHC. Current LHC data exclude deviations from the SM prediction [9-12] of more than about $15 \%$. (This limit, as well as the bounds discussed below, depends on a universal assumption about the absence of undetected Higgs decays which we will adopt for this paper.) For the couplings of type $g_{V, b 1}$, the bounds are weaker. For instance $\left(g_{V, b 1} \in[0.8,4.5]\right)$ was reported in ref. [9]. The measurement of double Higgs couplings $\operatorname{hhVV}\left(g_{V, a 2}\right.$ and $\left.g_{V, b 2}\right)$ is challenging at the LHC. Recently, ATLAS reported a search for double Higgs production in VBF [13], which excludes the ranges $g_{V, a 2}<-0.56$ and $g_{V, a 2}>2.89$.

At the LHC, searches for a double Higgs final state focus on the gluon-gluon fusion process $g g \rightarrow h h$. The Higgs decay channels $b \bar{b} b \bar{b}[14,15], b \bar{b} \gamma \gamma[16,17], b \bar{b} \tau \tau[18,19]$ and $b \bar{b} V V[20,21]$ have been investigated by both ATLAS and CMS. In addition, result for the 
channels $W W \gamma \gamma[22]$ and $W W W W[23]$ were reported by ATLAS. A combination of these searches can be found in refs. [24, 25]. It is shown that the Higgs self-coupling $\lambda_{3}$ can be constrained to $[-5,12]$ by data, while no constraint of $\kappa_{5}$ has been reported.

As a complementary process, double Higgs production via VBF at hadron colliders has been extensively studied in the literature [26-30]. The NLO QCD and higher order correction for this process has been calculated in refs. [31-36]; they find an enhancement of around $7 \%$ as it is natural for a pure electroweak process. For the high-luminosity LHC (HL-LHC), assuming $\mathcal{L}=3 \mathrm{ab}^{-1}$ at $14 \mathrm{TeV}$, it is expected that the couplings of type $g_{V, a 2}$ can be constrained to $20 \%$ [28], while a precision of around $1 \%$ should be achievable at a future $100 \mathrm{TeV}$ hadron collider [29]. Furthermore, the hhVV coupling is also accessible via $h V V$ or $h h V$ final states. Ref. [37] argues that a measurement of the $W^{ \pm} W^{ \pm} h$ final state can constrain the $h h W W$ coupling to $\mathcal{O}(100 \%)$ at the HL-LHC, and to $20 \%$ at a $100 \mathrm{TeV}$ collider, while the determination of this coupling from the $h h V$ final state can only yield a weak bound [38].

Possible measurements of the $g_{V, a 2}$ couplings, i.e., the Higgs interacting with the logitudinal components of massive vector bosons, have thus been covered in some detail in previous work. However, without further assumptions it is not evident that couplings of the Higgs to transversal vector bosons play a lesser role. In this work, we will aim at filling this gap and thus perform a Monte-Carlo study of the sensitivity to couplings of type $g_{V, b 2}$, both for the LHC and for future high-energy hadron colliders, and correlate this with the determination of $g_{V, a 2}$.

The VBF process $V V \rightarrow h h$ receives a contribution from the Higgs cubic self-coupling. It is well known that at hadron colliders, the Higgs self-coupling is most accessible in the gluon-gluon fusion process [39], whose cross section is one order of magnitude larger than that of the VBF process. This fact has received a lot of attention [40-64]. A measurement of the VBF process will provide additional precision for the Higgs self-coupling. However, for simplicity we will assume here that the couplings which are accessible in gluon-gluon fusion are known, and we fix those at their SM value. This allows us to focus on the couplings which are specific to the VBF class of processes, and lets us more easily estimate the sensitivity potential for those.

This paper is organized as follows. In section 2, we briefly introduce the mass-drop method used for tagging highly boosted Higgs bosons, and use it to explore the SM case at the LHC and at a $100 \mathrm{TeV}$ collider. In section 3, we investigate the potential for the discovery of new physics via multi-Higgs production, in form of the interactions discussed above. In section 4 , we provide projections for bounds on $g_{V, b 2}$ and $g_{V, a 2}$ for different collision energies. We conclude this paper with a discussion of our findings in section 5 .

\section{Higgs pair production in the Standard Model}

We will focus on the signal process $p p \rightarrow h h j j \rightarrow 4 b 2 j$ due to the reason that the decay channel $h \rightarrow b \bar{b}$ has the largest branch ratio. The partonic signal events are generated using WHIZARD [65] with the cuts listed in table 1. We takes the parton distribution functions from CTEQ611 [66]. 


\begin{tabular}{|c|c|c|c|}
\hline Cuts & $\sqrt{s}=14 \mathrm{TeV}$ & $\sqrt{s}=27 \mathrm{TeV}$ & $\sqrt{s}=100 \mathrm{TeV}$ \\
\hline$P_{t}(j)$ & $>20 \mathrm{GeV}$ & $>20 \mathrm{GeV}$ & $>30 \mathrm{GeV}$ \\
\hline$\Delta R(j, j)$ & $>0.8$ & $>0.8$ & $>0.8$ \\
\hline$|\eta(j)|$ & $<5.0$ & $<5.0$ & $<8.0$ \\
\hline$\Delta \eta\left(j_{1}, j_{2}\right)$ & $>3.6$ & $>3.6$ & $>4.0$ \\
\hline$m\left(j_{1}, j_{2}\right)$ & $>500 \mathrm{GeV}$ & $>500 \mathrm{GeV}$ & $>800 \mathrm{GeV}$ \\
\hline
\end{tabular}

Table 1. Acceptance cuts used for the calculation of VBF Higgs production in $p p$ collision (VBF cuts), for three different collider energies. The $j_{1}$ and $j_{2}$ are the tagged forward jets for VBF process.

In this work, we consider the main backgrounds $p p \rightarrow t \bar{t} \rightarrow 2 b 4 j, p p \rightarrow 2 b 4 j$ (QCD) and $p p \rightarrow 4 b 2 j$. The background events $p p \rightarrow t \bar{t} \rightarrow 2 b 4 j$ are generated by WHIZARD. We have cross-checked the results with Madgraph [67]. Pure-QCD partonic events of type $p p \rightarrow 2 b 4 j$ and $p p \rightarrow 4 b 2 j$ are generated using ALPGEN [68].

For all event samples, parton shower and hadronization are performed by Pythia 8 [69]. Jets are reconstructed by FastJet [70] using the anti- $k_{t}$ algorithm [71] with a jet radius $R=0.4$ and transverse momentum cut $P_{t}>20 \mathrm{GeV}$. We do not account for detector effects in detail but insert values for efficiency and mistagging rates where appropriate.

\subsection{Analysis method at the $14 \mathrm{TeV}$ LHC}

In table 2 (first column) we list the expected number of signal and background events in the SM for the LHC with $\sqrt{s}=14 \mathrm{TeV}$ and luminosity $\mathcal{L}=3 \mathrm{ab}^{-1}$. To suppress the QCD background, we require four b-tagged jets in the final state $\left(n_{b}=4\right)$. We assume the b-tagging efficiency $\epsilon_{b}=0.7$ and mistagging rate $\epsilon_{\text {miss }}=0.001$. The number of events after applying the b-tagging requirement are listed in the 2 nd column of table 2 .

To identify two forward jets in the VBF process, we first select the jet with highest energy and label it as $j_{1}$. If its energy satisfies $E_{j_{1}}>500 \mathrm{GeV}$, we scan over all other jets and determine the maximal rapidity difference $\Delta \eta\left(j_{1}, j\right)$ and invariant mass $m\left(j_{1}, j\right)$ with respect to the leading jet. If the conditions $\Delta \eta\left(j_{1}, j\right)_{\max }>3.6$ and $m\left(j_{1}, j\right)_{\max }>500 \mathrm{GeV}$ are met simultaneously, we identify the most energetic $\mathrm{j}$ as $j_{2}$ and label corresponding pair of jets as the tagging forward jets of a VBF process. Otherwise, the event is rejected. The number of events after applying this VBF cut are listed in the 3rd column of table 2.

With these b-jet and forward-jet tagging requirements, the backgrounds $p p \rightarrow t \bar{t}$ and $p p \rightarrow 2 b 4 j$ are greatly reduced. The dominant remaining background originates from QCD processes $p p \rightarrow 4 b 2 j$, where the cross section after cuts is still five orders of magnitude larger than that of the signal.

To further suppress this huge background, we select those events with massive jets formed by highly boosted Higgs bosons and adopt the mass drop method [72].

\subsubsection{The mass drop method for highly-boosted Higgs boson tagging}

A significant fraction (of the order of $10 \%$ ) of the VBF event sample in the SM contains highly boosted Higgs bosons. A highly boosted Higgs boson has a large transverse momen- 


\begin{tabular}{|c|ccc|}
\hline Process & $\sigma \times \mathcal{L}$ & $n_{b}=4$ & VBF \\
\hline SM signal & 993 & 238 & 171 \\
\hline$p p \rightarrow 4 b 2 j$ & $2.28 \times 10^{8}$ & $5.47 \times 10^{7}$ & $1.86 \times 10^{7}$ \\
$p p \rightarrow 2 b 4 j(\mathrm{QCD})$ & $2.38 \times 10^{10}$ & $1.14 \times 10^{4}$ & $3.85 \times 10^{4}$ \\
$p p \rightarrow t \bar{t} \rightarrow 2 b 4 j$ & $7.89 \times 10^{8}$ & 387 & 58 \\
\hline
\end{tabular}

Table 2. The cut efficiencies of b-tagging and VBF at $14 \mathrm{TeV}$ LHC are demonstrated. The total integrated luminosity is assumed to be $\mathcal{L}=3000 \mathrm{fb}^{-1}$. The b-tagging efficiency is $\epsilon_{b}=0.7$, and the mis-tagging rate of light quarks is $\epsilon_{\text {miss }}=0.001$.

tum $\left(P_{t}>200 \mathrm{GeV}\right)$ and can be detected in the central region of the detector. The decay products of the Higgs boson typically form a fat jet with a large jet mass, if a large cone parameter is used for the analysis. A fraction of the QCD background events also contains massive jets, but the fat jets originating from Higgs pairs can in principle be distinguished by their characteristic jet sub-structure.

In recent years, various methods for jet substructure analysis have been developed: (1) jet-grooming methods aim at removing soft radiation which is unlikely to originate from the hard process (see, e.g., [72]). (2) Radiation-constraint methods impose a cut on jet shape to separate the signal from the background (see, e.g., [73]). (3) Prong-finder methods detect a massive boosted object as a fat jet with multiple hard cores by exploiting the recombination history of the jet algorithm. As a particular prong-finder algorithm, the mass-drop tagger method is particularly suited for isolating boosted Higgs bosons, decaying to $b \bar{b}$, from the QCD background [72]. A detail review of jet substructure can be found in ref. [74]

In this work, we adopt the mass-drop tagger [72] as a means for tagging highly boosted Higgs bosons in the final state of the VBF process. The method consists of the following two steps: (1) we first identify jets by the standard anti- $k_{t}$ algorithm with the cone parameter $R=0.4$. After identifying the forward jets associated with the VBF process, we recluster the remaining jets using the Cambridge/Aachen (CA) algorithm $[75,76]$ with $R=1.2$. (2) If there are 2 to $4 \mathrm{CA}$ jets in an event, and its leading or subleading jet has a transverse momentum satisfying $P_{t}>200 \mathrm{GeV}$ and a jet mass $m_{j}>100 \mathrm{GeV}$, we apply the following procedure to tag candidates for highly boosted Higgs bosons.

1. Undo the last step of clustering of jet $j$ to get two daughter jets $j_{1}$ and $j_{2}$ with $m_{j 1}>m_{j 2}$.

2. If the conditions

$$
m_{j 1}<\mu m_{j} \quad \text { and } \quad \frac{\min \left(P_{t}\left(j_{1}\right), P_{t}\left(j_{2}\right)\right)}{m_{j}^{2}} \Delta R_{j 1, j 2}^{2}>y_{\text {cut }}
$$

are satified, we identify $j$ as a fat jet associated with a highly boosted Higgs boson.

3. Otherwise, we redefine $j$ as $j_{1}$ and repeat the above procedure. 


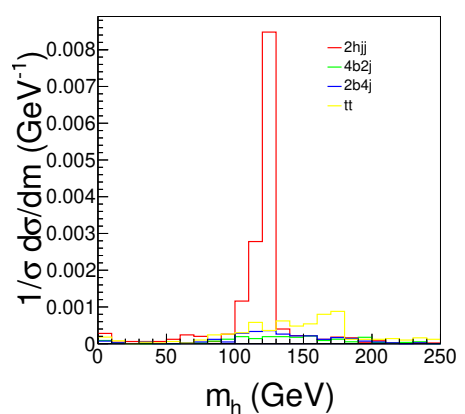

(a) $2 \mathrm{BH}$.

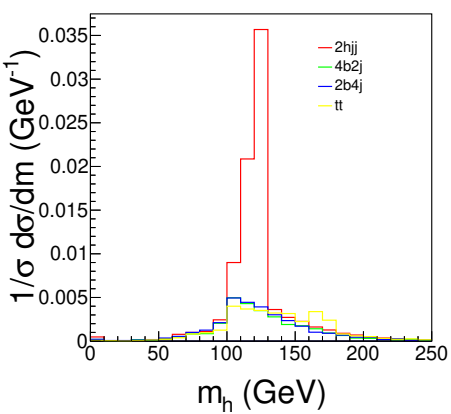

(b) $1 \mathrm{BH}$.

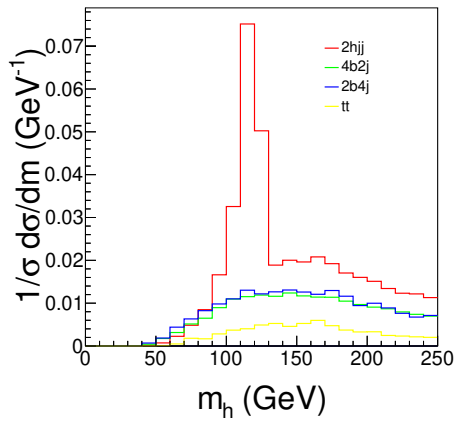

(c) $0 \mathrm{BH}$.

Figure 1. Distributions of the reconstructed Higgs mass with (a) 2-boosted Higgs events, (b) 1-boosted Higgs events, and (c) 0-boosted Higgs events.

There are two dimensionless parameters $\mu$ and $y_{\text {cut }}$ in this method, as given in eq. (2.1). In this work, we fix them as $\mu=0.67$ and $y_{\text {cut }}=0.09$. After two subjets are found, we also apply the filtering method to remove soft radiation which originates from the underlying event and contaminates $\mathrm{CA}$ jets with a larger cone size.

After applying this tagging algorithm, both signal and background events fall into three classes: 2-boosted Higgs (2BH), 1-boosted Higgs (1BH) and 0-boosted Higgs (0BH) candidate events. The number of events for each class are listed in table 3.

We observe that the fraction of signal events in the $2 \mathrm{BH}$ category is still small $(2-3 \%)$. Nevertheless, the corresponding background is two orders of magnitude lower than for the $0 \mathrm{BH}$ category, where the fraction of signal events is even less. The $1 \mathrm{BH}$ category falls in between. The tagger significantly improves the chance for finding signal events, but by itself it is clearly not sufficient for a measurement if the rate is SM-like.

In order to construct kinematic observables which improve the signal vs. background discrimination, we can reconstruct the mass peaks of the Higgs bosons for each event category. For each of the $2 \mathrm{BH}$ events, two jet masses $m_{j}$ should peak around Higgs the boson mass $m_{h}$, as shown in figure 1 . For a $1 \mathrm{BH}$ event, the jet mass of the leading fat jet should peak around the mass of the Higgs boson, while the mass of the second reconstructed Higgs candidate should coincide with the invariant mass of two b jets. For a 0BH event, two Higgs boson candidates are reconstructed by using the $\chi^{2}$ method. To this end, we define $\chi^{2}$ as follows:

$$
\chi^{2}(m)=\frac{\left|m\left(j_{1}, j_{2}\right)-m_{h}\right|^{2}}{\sigma_{j}^{2}}+\frac{\left|m\left(j_{3}, j_{4}\right)-m_{h}\right|^{2}}{\sigma_{j}^{2}}
$$

We assume $m_{h}=125 \mathrm{GeV}$, and $m\left(j_{1}, j_{2}\right)$ and $m\left(j_{3}, j_{4}\right)$ are the invariant mass of two jet pairs in the final state, scanning over each combination. $\sigma_{j}=10 \mathrm{GeV}$ is used to take into account the error in jet energy resolution. The pairing which minimizes $\chi^{2}$ is selected, and the corresponding invariant masses determined by the pairs of jets are taken as the reconstructed masses of Higgs bosons. 


\begin{tabular}{|c|ccc|}
\hline & $\begin{array}{c}\text { 2-boosted Higgs } \\
(2 \mathrm{BH})\end{array}$ & $\begin{array}{c}\text { 1-boosted Higgs } \\
(1 \mathrm{BH})\end{array}$ & $\begin{array}{c}\text { 0-boosted Higgs } \\
(0 \mathrm{BH})\end{array}$ \\
\hline SM Signal & 4 & 21 & 146 \\
\hline$p p \rightarrow 4 b 2 j$ & $1.17 \times 10^{5}$ & $1.56 \times 10^{6}$ & $1.69 \times 10^{7}$ \\
$p p \rightarrow 2 b 4 j(\mathrm{QCD})$ & 28 & 349 & $3.81 \times 10^{4}$ \\
$p p \rightarrow t \bar{t} \rightarrow 2 b 4 j$ & 3 & 13 & 42 \\
\hline
\end{tabular}

Table 3. The numbers of events in the $2 \mathrm{BH}$ case, $1 \mathrm{BH}$ case and $0 \mathrm{BH}$ case at $14 \mathrm{TeV}$ LHC are tabulated.

We display the distributions of the reconstructed mass of the leading Higgs boson in figure 1 . The shapes of the $2 \mathrm{BH}$ and $1 \mathrm{BH}$ cases are almost identical. We note that the Higgs peak in the $2 \mathrm{BH}$ case is narrower than in the $0 \mathrm{BH}$ case, since wrong pairings are rejected more efficiently.

\subsubsection{Multivariate analysis}

Results in table 3 clearly show the challenge in the signal/background separation. Even when a mass cut on the tagged massive jet is imposed (say $\left|m_{h^{0}}-125\right|<25 \mathrm{GeV}$ ), the number of the background events of processes $4 \mathrm{~b} 2 \mathrm{j}$ is only suppressed by a factor 10 . To further improve the ratio of signal over backgrounds after the VBF cuts and boosted Higgs taggings, we need more advanced method to optimize cuts.

Traditionally, distributions of various kinematic observables of signal and background are studied, and for some selected and uncorrelated observables, a few single cuts can be imposed to separate signal/background. Such a method is sufficient for some simple processes where distributions of the signals and backgrounds are situated in the feature space significantly different. But it becomes far from optimal for some more complicated processes where the dimensionality of feature space for final states are high and large number of observables needed to be reconstructed and introduced as one of the dimensions of the feature space, like the case of full hadronic states of top quark pair production at hadron colliders which have been demonstrated to be successful.

Instead of introducing a cut on each single observables sequentially, in the multivariate analysis (MVA), one event is viewed as a point in a high dimensional feature space defined by the chosen/reconstructed observables. Typically, the signal and background events are assumed to situated at different regions of feature space. Thus the traditional cut-based method is equivalent to choosing hypersurfaces or hypercubes to separate signal/background events. Moreover, the modern multivariate analysis can choose any kind of such subregions, even disconnected, so as to reach the optimal discriminant of signal and background events.

In practice, finding subregions enriched with signal/background events in high-dimensional feature space is a challenging task, and thus various computer algorithms are designed to accomplish this mission. The most successful algorithms for classification are based on the (supervised) machine learning. In this kind of algorithm, a sufficient number of signal 
and background data samples is supplied at the every beginning, so that the algorithm can know (or "learn") where signal/background events situate in the feature space. After this training, it can use the learnt information and can evaluate an event to be signal-like or background-like. It should be emphasised that to guarantee the success of signal and background discrimination, sufficient observables should be introduced in order to describe the whole feature space of signal/background. Meanwhile the training data sample should be large enough to cover most of the subregions of signal/background events.

Machine learning algorithms have been introduced into high-energy physics many years ago [77], and today they play many important roles in experimental and phenomenological works, from low-level tracking and triggers, to high-level object reconstructions and analysis, and the most common signal-background discriminations. Among those algorithms, the boosted decision trees (BDT) with the adaptive boost algorithm [78] is often referred as the best out-of-the-box classifier. One decision tree is similar to the traditional cut-based method: it consists multiple steps, and in each step a cut is set on one observable. Such a method is simple and can capture the most important features of signal and backgrounds. To further improve its performance, the adaptive boost algorithm is proposed, where subsequent decision trees are trained with a particular attention to the events mis-classified by previous decision trees. In other words, the BDT divides the feature space for both signal and background into hypercubes and identify the signal/background enriched subregions. As a result, the combination of all decision trees can produce a much better results.

Since the method BDT is a MVA depending upon nonlinear transformation of variables of feature space, it utilizes the fact that the correlations of feature variables are different for signal and background events so that they can situate at different subregions of feature space. One of its advantages is that its output is always stable and resilient even when two observables are identical. Another remarkable advantage is that it is fast when compared with the neural network method with the same number of inputs.

We adopt BDT implementation in TMVA [79] to optimize cuts of feature space. In particular, we select the following observables as the input to our BDT analysis:

- $P_{t}\left(h_{i}\right)$ : the transverse momenta of the two reconstructed Higgs bosons.

- $m\left(h_{i}\right)$ : the invariant masses of the reconstructed Higgs bosons.

- $P_{t}\left(j_{i}\right)$ : the transverse momenta of the two forward jets.

- $E\left(j_{i}\right)$ : the energies of the forward jets.

- $\eta\left(j_{i}\right)$ : the pseudo-rapidity of the forward jets.

- $m(j, j)$ : the invariant mass of the forward jets.

- $\Delta \eta(j, j)$ : the rapidity difference of the forward jets.

- $P_{t}\left(j_{i}^{\text {sub }}\right)$ : the transverse momenta of the two subjets of each highly boosted Higgs boson.

- $m(h, h)$ : the invariant mass of the two Higgs boson candidates.

- $\chi_{\min }^{2}$ (only for the $0 \mathrm{BH}$ case): the minimum value of $\chi^{2}$. 


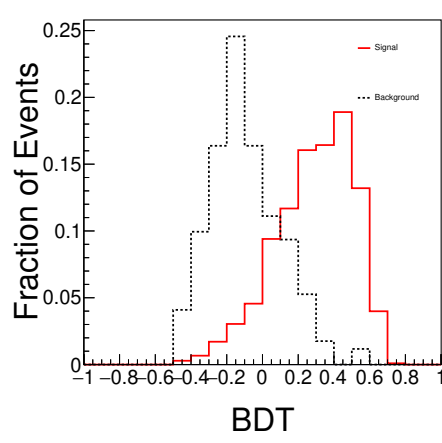

(a) 2-boosted Higgs.

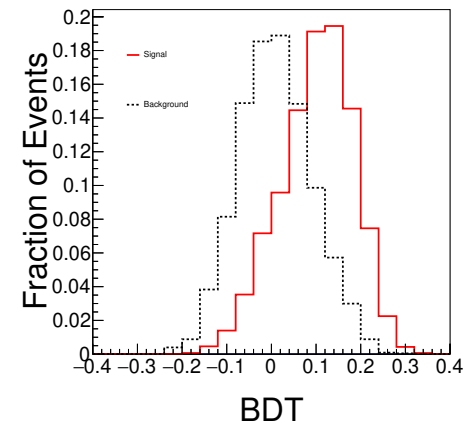

(b) 1-boosted Higgs.

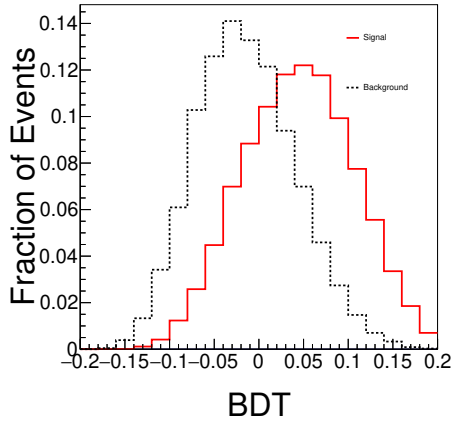

(c) 0-boosted Higgs.

Figure 2. The response of the discriminants to the SM signal and background at $14 \mathrm{TeV}$ LHC with (a) 2-boosted events, (b) 1-boosted events, and (c) 0-boosted events.

\begin{tabular}{|c|ccc|}
\hline & $\begin{array}{c}\text { 2-boosted Higgs } \\
(2 \mathrm{BH})\end{array}$ & $\begin{array}{c}\text { 1-boosted Higgs } \\
(1 \mathrm{BH})\end{array}$ & $\begin{array}{c}\text { 0-boosted Higgs } \\
(0 \mathrm{BH})\end{array}$ \\
\hline SM Signal with BDT cut & 3 & 13 & 90 \\
\hline Background with BDT cut & $2.06 \times 10^{4}$ & $3.05 \times 10^{5}$ & $4.42 \times 10^{6}$ \\
\hline$S / B$ & $1.40 \times 10^{-4}$ & $4.30 \times 10^{-5}$ & $2.04 \times 10^{-5}$ \\
\hline$S / \sqrt{S+B}$ & 0.020 & 0.024 & 0.043 \\
\hline
\end{tabular}

Table 4. The significances of the BDT at $14 \mathrm{TeV}$ LHC are demonstrated.

The results of the BDT response are presented in the figure 2. Obviously, signal and background can separated best in the $2 \mathrm{BH}$ case. For the $1 \mathrm{BH}$ and $0 \mathrm{BH}$ cases, extracting the signal is challenging even after exploiting the BDT method.

We can optimize the BDT cut to achieve the maximal significance, which is defined as $S / \sqrt{S+B}$, where $S$ is the event number of the signal and $B$ is the event number of the total background. The efficiencies and significances of the optimized BDT cut are listed the table 4 for all three event classes.

Comparing the results given in table 3 and table 4, we conclude that the BDT reduces the background by one additional order of magnitude, improving on the sequential cut method. Nevertheless, the final number of SM signal events is still tiny compared to the background in all cases, and the significance can only reach $0.02(2 \mathrm{BH}, 1 \mathrm{BH})$ or $0.04(0 \mathrm{BH})$. This is still far from the requirement of a discovery at the LHC.

\subsection{Analysis of $p p \rightarrow h h j j$ in SM at $100 \mathrm{TeV}$ hadron collider}

We now apply the methods as described above to the VBF process at a future $100 \mathrm{TeV}$ hadron collider. We assume a high integrated luminosity of $\mathcal{L}=30 \mathrm{ab}^{-1}$, and adapt all selection parameters to the different environment as appropriate.

We require that the most energetic jet has an energy $E_{j}>800 \mathrm{GeV}$. The VBF cuts are adjusted as $\Delta \eta_{j j \text {, max }}>4.0$ and $m_{j j, \max }>800 \mathrm{GeV}$. Table 5 shows the number of events 


\begin{tabular}{|c|ccc|}
\hline Process & $\sigma \times \mathcal{L}$ & $n_{b}=4$ & VBF \\
\hline SM signal & $4.28 \times 10^{5}$ & $1.03 \times 10^{5}$ & $8.96 \times 10^{4}$ \\
\hline$p p \rightarrow 4 b 2 j$ & $5.02 \times 10^{10}$ & $1.21 \times 10^{10}$ & $8.51 \times 10^{9}$ \\
$p p \rightarrow 2 b 4 j$ & $5.04 \times 10^{12}$ & $2.47 \times 10^{6}$ & $1.83 \times 10^{6}$ \\
$p p \rightarrow t \bar{t} \rightarrow 2 b 4 j$ & $3.93 \times 10^{11}$ & $1.93 \times 10^{5}$ & $6.20 \times 10^{4}$ \\
\hline
\end{tabular}

Table 5. The cut efficiencies of b-tagging and VBF at $100 \mathrm{TeV}$ collider are demonstrated. Here, the total integrated luminosity is assumed to be $\mathcal{L}=30 \mathrm{ab}^{-1}$. b-tagging efficiency is $\epsilon_{b}=0.7$, and miss tagging rate is $\epsilon_{\text {miss }}=0.001$.

\begin{tabular}{|c|ccc|}
\hline & $\begin{array}{c}\text { 2-boosted Higgs } \\
(2 \mathrm{BH})\end{array}$ & $\begin{array}{c}\text { 1-boosted Higgs } \\
(1 \mathrm{BH})\end{array}$ & $\begin{array}{c}\text { 0-boosted Higgs } \\
(0 \mathrm{BH})\end{array}$ \\
\hline SM Signal & 4265 & $1.76 \times 10^{4}$ & $6.77 \times 10^{4}$ \\
\hline$p p \rightarrow 4 b 2 j$ & $3.65 \times 10^{8}$ & $2.01 \times 10^{9}$ & $6.13 \times 10^{9}$ \\
$p p \rightarrow 2 b 4 j$ & $8.35 \times 10^{4}$ & $4.40 \times 10^{5}$ & $1.31 \times 10^{6}$ \\
$p p \rightarrow t \bar{t} \rightarrow 2 b 4 j$ & 8244 & $2.20 \times 10^{4}$ & $3.18 \times 10^{4}$ \\
\hline
\end{tabular}

Table 6. The numbers of events for $2 \mathrm{BH}, 1 \mathrm{BH}$ and $0 \mathrm{BH}$ cases at $100 \mathrm{TeV}$ collider are tabulated.

after imposing b-tagging and VBF cuts. Due to the increased cross section at high energy and the high luminosity of the collider, the number of signal events is increased by a factor 1000 compared to the high-luminosity LHC. After applying b-tagging and VBF cuts, the total number of the signal events is $8.96 \times 10^{4}$. Of course, the number of background events also increases and reaches $10^{9}$, so background reduction is still essential.

The number of events for the $2 \mathrm{BH}, 1 \mathrm{BH}$ and $0 \mathrm{BH}$ cases are listed in table 6 . As one would expect, more events with highly boosted Higgs bosons can be observed at the $100 \mathrm{TeV}$ collider than at the LHC. This is illustrated by the SM curves of figure 4. To enable signal/background discrimination in these three cases, we apply the BDT method as described above. The results are shown in figure 3 and table 7 . We can set the BDT cut in a region where the residual background is effectively zero.

These results show that it is possible in principle to discover the SM signal at a $100 \mathrm{TeV}$ collider. The large rate of the VBF process at high collision energy leaves enough signal events after all measures for background reduction have been applied. We note, however, that pileup effects in a high luminosity run might pose a challenge for analyses such as this one. For a final verdict on the detectability of the SM signal, a more sophisticated full-simulation study would be necessary which is beyond the scope of this paper.

\section{Multi-Higgs production in VBF processes with dimension-6 operators}

In this section, we extend our study of the $p p \rightarrow h h j j$ process to contributions beyond the SM. Given the phenomenological Lagrangian (1.3), such effects are parameterized in terms of the coefficients $g_{i}$, where we focus in particular on the $g_{V, b 2}$ coupling that describes a 


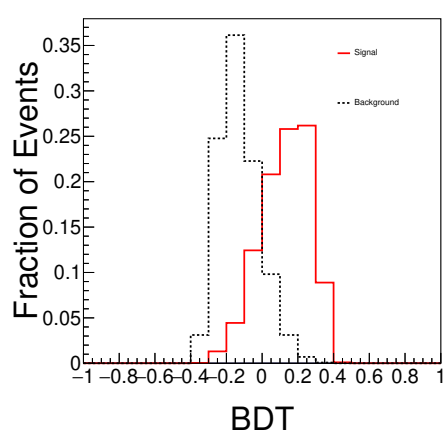

(a) $2 \mathrm{BH}$.

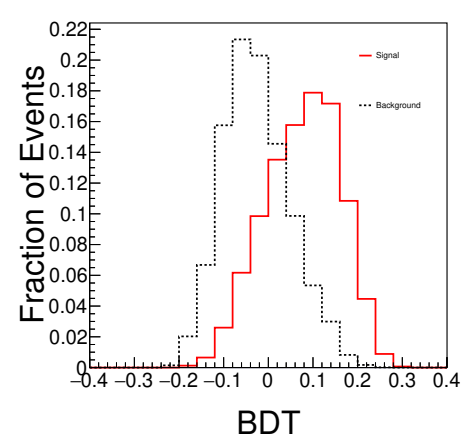

(b) $1 \mathrm{BH}$

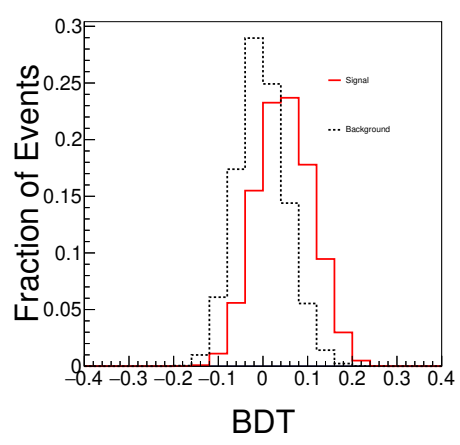

(c) $0 \mathrm{BH}$.

Figure 3. The response of the discriminants to the SM signal and background at $100 \mathrm{TeV}$ collider with (a) 2BH case, (b) 1BH case, and (c) $0 \mathrm{BH}$ case.

\begin{tabular}{|c|ccc|}
\hline & $\begin{array}{c}\text { 2-boosted Higgs } \\
(2 \mathrm{BH})\end{array}$ & $\begin{array}{c}\text { 1-boosted Higgs } \\
(1 \mathrm{BH})\end{array}$ & $\begin{array}{c}\text { 0-boosted Higgs } \\
(0 \mathrm{BH})\end{array}$ \\
\hline SM Signal with BDT cut & 298 & 12 & 90 \\
\hline Background with BDT cut & 0 & 0 & 78 \\
\hline$S / B$ & - & - & 1.15 \\
\hline$S / \sqrt{S+B}$ & 17.27 & 3.43 & 6.923 \\
\hline
\end{tabular}

Table 7. The significances of the BDT at $100 \mathrm{TeV}$ collider are demonstrated.

non-SM double-Higgs interaction with the transverse polarization components of the vector bosons.

\subsection{Effective-theory description}

If we introduce the gauge symmetry of the SM in the phenomenological description, any anomalous effects can be re-expressed in terms of higher-dimensional gauge-invariant operators. To avoid redundancy, it is convenient to choose a particular operator basis such as the SILH basis [80] that we adopt for this work. As usual, we truncate the power-series expansion of the gauge-invariant effective theory at dimension six. This truncation allows us to express the phenomenological couplings in (1.3) in terms of a small number of SILH operator coefficients. Such a simplification allows for relating quantitative results for the VBF Higgs-pair process to the analysis of existing data, as well as to studies of different processes and interactions.

We list the relevant terms in the SILH basis,

$$
\begin{aligned}
\mathcal{L}_{\mathrm{SILH}} \supset & \frac{i c_{W} g}{2 m_{\rho}^{2}}\left(H^{\dagger} \sigma^{i} \overleftrightarrow{D^{\mu}} H\right)\left(D^{\nu} W_{\mu \nu}\right)^{i}+\frac{i c_{B} g^{\prime}}{2 m_{\rho}^{2}}\left(H^{\dagger} \overleftrightarrow{D^{\mu}} H\right)\left(\partial^{\nu} B_{\mu \nu}\right) \\
& +\frac{i c_{H W} g}{16 \pi^{2} f^{2}}\left(D^{\mu} H\right)^{\dagger} \sigma^{i}\left(D^{\nu} H\right) W_{\mu \nu}^{i}+\frac{i c_{H B} g^{\prime}}{16 \pi^{2} f^{2}}\left(D^{\mu} H\right)^{\dagger}\left(D^{\nu} H\right) B_{\mu \nu} \\
& +\frac{c_{\gamma} g^{\prime 2}}{16 \pi^{2} f^{2}} \frac{g^{2}}{g_{\rho}^{2}} H^{\dagger} H B_{\mu \nu} B^{\mu \nu} .
\end{aligned}
$$


In the following, we make use of the result from $[4,81]$ in table 8 which relates the phenomenological coefficients such as $g_{V, b}$ to the parameters of (3.1). In particular, the couplings $g_{V, b}$ that we are interested in, only depend on $c_{H W}, c_{H B}$ and $c_{\gamma}$, but not on $c_{W}$ and $c_{B}$ which determine $g_{V, a}$. The SILH parameterization has been evaluated as the low-energy limit of various ultra-violet theories, such as models where the Higgs becomes a pseudo Nambu-Goldstone boson theory or holographic completions with extra dimensions; cf. [58]. We note the correlations between $g_{V, a 1}$ and $g_{V, a 2}, g_{V, b 1}$ and $g_{V, b 2}$ which follow from the dimension-six truncation, and should be tested against real data if actual deviations from the SM show up. The SILH was derived firstly from various strongly interacting Higgs scenarios, however it can be as general as Warsaw basis with a clear UV picture. For example, a recent study with vector-like fermion extension [82] can also be a type of UV theories for two parameters $g_{V, b}$ and $g_{V, a}$, especially we can consider the vector-like fermions as the top-partners (quark-partners) in some versions of little Higgs.

In the SILH effective Lagrangian, Higgs interactions include further dimension-six operators such as

$$
\frac{c_{H}}{2 f^{2}} \partial^{\mu}\left(H^{\dagger} H\right) \partial_{\mu}\left(H^{\dagger} H\right)+\frac{c_{T}}{2 f^{2}}\left(H^{\dagger} \overleftrightarrow{D^{\mu}} H\right)\left(H^{\dagger} \overleftrightarrow{D}_{\mu} H\right)
$$

The coefficients of these operators are switched off in our analysis because they are to be measured in different processes. In particular, the first one entails a global shift to all Higgs interactions which is equivalent to a modified Higgs total width, while the latter violates the custodial symmetry of weak interactions and globally modifies $Z Z$ vs. $W W$ Higgs couplings. In our current work we assume that no custodial-symmetry violation beyond the $\mathrm{SM}$ is present, as detailed below.

In the third column of table 8 , we present some typical numerical values for these parameters. Considering $g \sim 0.654, g^{\prime} \sim 0.350, v \sim 246 \mathrm{GeV}, \tan \theta=g^{\prime} / g=0.535$, $\alpha=\frac{g^{2} v^{2}}{32 \pi^{2} f^{2}}=8.2 \times 10^{-5}$ with $f=1 \mathrm{TeV}$ and $g_{\rho} \sim \frac{4 \pi}{\sqrt{3}}, m_{\rho}=g_{\rho} f=7.3 \mathrm{TeV}$, we can simplify the expressions in this table with

$$
\begin{aligned}
\zeta_{h} & \sim 1, \quad \zeta_{A} \sim 1, \quad y_{Z A} \sim \alpha \frac{g^{\prime}}{g}\left(c_{H W}-c_{H B}\right) \\
\zeta_{Z} & \sim 1-\frac{1}{8} \alpha^{2}\left(\frac{g^{\prime}}{g}\right)^{2}\left(c_{H W}-c_{H B}\right)^{2}, \quad \zeta_{A Z} \sim \alpha \frac{g^{\prime}}{4 g}\left(c_{H W}-c_{H B}\right), \quad \zeta_{W} \sim 1-\frac{\alpha}{2} c_{H W} .
\end{aligned}
$$

(Our notation slightly differs from the definitions for $\bar{c}_{i}$ used in ref. [83].) To simplify our discussion on the future collider sensitivity on $g_{V, b 2}$ and $g_{V, a 2}$, we assume custodialsymmetry relations, namely that $g_{W, b 2}=g_{Z, b 2}$ and $g_{X, b 2}=g_{A, b 2}=0$ and $g_{W, a 2}=g_{Z, a 2}$. This roughly renders $c_{\gamma}, c_{H B}, c_{B} \sim 0$, so we can perform a two-parameter study on $c_{W}$ and $c_{H W}$. The interactions proportional to $g_{W, b 2}$ and $g_{W, a 2}\left(g_{Z, b 2}\right.$ and $\left.g_{Z, a 2}\right)$ account for dominant contributions to the cross section of $p p \rightarrow h h j j$ process, up to $70 \%$ (30\%), respectively. 


\begin{tabular}{|c|c|c|}
\hline & SILH & numerics with assumptions below \\
\hline$g_{W, b 1}$ & $c_{H W} \frac{g^{2} v^{2}}{32 \pi^{2} f^{2}} \zeta_{h} \zeta_{W}^{2}$ & $10^{-4} c_{H W}$ \\
\hline$g_{W, b 2}$ & $g_{W, b 1} \zeta_{h}$ & $10^{-4} c_{H W}$ \\
\hline$g_{A, b 1}$ & $-c_{\gamma} \frac{g^{2} v^{2}}{8 \pi^{2} f^{2}} \frac{g^{\prime 2}}{g_{\rho}^{2}} \cos ^{2} \theta \zeta_{h} \zeta_{A}^{2}$ & $-10^{-6} c_{\gamma}$ \\
\hline$g_{A, b 2}$ & $g_{A, b 1} \zeta_{h}$ & $-10^{-6} c_{\gamma}$ \\
\hline$g_{X, b 1}$ & $\frac{g g^{\prime} v^{2}}{64 \pi^{2} f^{2}}\left[\left(c_{H W}-c_{H B}\right)+8 c_{\gamma} \frac{g^{2}}{g_{\rho}^{2}} \sin ^{2} \theta\right] \zeta_{h} \zeta_{A} \zeta_{Z}$ & $10^{-5}\left(c_{H W}-c_{H B}\right)$ \\
\hline$g_{X, b 2}$ & $+c_{\gamma} \frac{g^{2} v^{2}}{4 \pi^{2} f^{2}} \frac{g^{\prime 2}}{g_{\rho}^{2}} \cos ^{2} \theta \zeta_{h} \zeta_{A Z}^{2}$ & $10^{-5}\left(c_{H W}-c_{H B}\right)$ \\
\hline$g_{Z, b 1}$ & $g_{X, b 1} \zeta_{h}$ & $10^{-4}\left(c_{H W}+0.29 c_{H B}\right)$ \\
\hline$g_{Z, b 2}$ & $-\frac{g g^{\prime} v^{2}}{64 \pi^{2} f^{2}}\left[\left(c_{H W}-c_{H B}\right)+8 c_{\gamma} \frac{g^{2}}{g_{\rho}^{2}} \sin ^{2} \theta\right] \zeta_{h} \zeta_{A Z} \zeta_{Z}$ & $10^{-4}\left(c_{H W}+0.29 c_{H B}\right)$ \\
\hline$g_{W, a 1}$ & {$\left[1-\left(c_{W} \frac{g^{2} v^{2}}{m_{\rho}^{2}}+c_{H W} \frac{g^{2} v^{2}}{16 \pi^{2} f^{2}}\right)\right] \zeta_{h} \zeta_{W}^{2}$.} & $1-2 \times 10^{-4}\left(3 c_{W}+c_{H W}\right)$ \\
\hline$g_{W, a 2}$ & {$\left[1-3\left(c_{W} \frac{g^{2} v^{2}}{m_{\rho}^{2}}+c_{H W} \frac{g^{2} v^{2}}{16 \pi^{2} f^{2}}\right)\right] \zeta_{h}^{2} \zeta_{W}^{2}$} & $1-6 \times 10^{-4}\left(3 c_{W}+c_{H W}\right)$ \\
\hline$g_{Z, a 1}$ & {$\left[1-\left(c_{W} \frac{g^{2} v^{2}}{m_{\rho}^{2}}+c_{B} \frac{g^{\prime 2} v^{2}}{m_{\rho}^{2}}+c_{H W} \frac{g^{2} v^{2}}{16 \pi^{2} f^{2}}+c_{H B} \frac{g^{\prime 2} v^{2}}{16 \pi^{2} f^{2}}\right)\right] \zeta_{h} \zeta_{Z}^{2}$} & $1-2 \times 10^{-4}\left[3\left(c_{W}+0.29 c_{B}\right)+c_{H W}+0.29 c_{H B}\right]$ \\
\hline$g_{Z, a 2}$ & {$\left[1-3\left(c_{W} \frac{g^{2} v^{2}}{m_{\rho}^{2}}+c_{B} \frac{g^{\prime 2} v^{2}}{m_{\rho}^{2}}+c_{H W} \frac{g^{2} v^{2}}{16 \pi^{2} f^{2}}+c_{H B} \frac{g^{\prime 2} v^{2}}{16 \pi^{2} f^{2}}\right)\right] \zeta_{h}^{2} \zeta_{Z}^{2}$} & $1-6 \times 10^{-4}\left[3\left(c_{W}+0.29 c_{B}\right)+c_{H W}+0.29 c_{H B}\right]$ \\
\hline
\end{tabular}

Table 8. Relations between the phenomenological Lagrangian parameters in (1.1)-(1.3) (first column), the SILH effective Lagrangian (3.1) (second column), The extra parameters $\zeta_{h}^{n}, \zeta_{W}^{n}, \zeta_{Z}^{n}$, $\zeta_{A}^{n}, \zeta_{A Z}^{n}$ (defined in [4, 81]) are induced by the Higgs and gauge-boson wave-function normalization, respectively.

\subsection{Higgs-pair couplings to transverse vector polarizations in VBF}

Introducing non-SM effects proportional to the $g_{V, b 2}$ couplings, we consider kinematical distributions and their discriminating power. In figure 4, we display the distributions of the $P_{t}$ of the leading Higgs boson and the invariant mass the Higgs-bosons pair, respectively. The subfigures $4 \mathrm{a}$ and $4 \mathrm{~b}$ show the distributions at the LHC with collision energy $14 \mathrm{TeV}$. The new-physics effect is illustrated by the green and blue curves which correspond to two different values of $g_{V, b 2}$, namely $g_{V, b 2}=0.09$ and $g_{V, b 2}=0.18$, repectively. We observe a huge enhancement at high $P_{t}$ in the curves which include the new interaction of Higgs bosons with transversal gauge bosons. For the selected parameter values, the fraction of events in the region with $P_{t}>200 \mathrm{GeV}$ increases to $50 \%$ and $70 \%$, respectively, while in the SM this fraction is just $18 \%$.

The corresponding distributions at a $100 \mathrm{TeV}$ hadron collider are shown in the figure $4 \mathrm{c}$ and $4 \mathrm{~d}$, where we select $g_{V, b 2}=0.018$ and 0.024 . In this case, the fraction of events in the region with $P_{t}>200 \mathrm{GeV}$ increases to $50 \%$ and $60 \%$, respectively, while in the SM, it is $25 \%$.

Subfigures $4 \mathrm{~b}$ and $4 \mathrm{~d}$ contain the Higgs-pair invariant-mass distributions for the LHC and for the $100 \mathrm{TeV}$ collider, which are likewise enhanced in the high-mass region if anomalous effects are included.

As a concrete numerical example for the analysis in presence of new-physics effects, in table 9 we demonstrate the cut flow for the value $g_{V, b 2}=0.18$ at the $14 \mathrm{TeV} \mathrm{LHC}$. For 


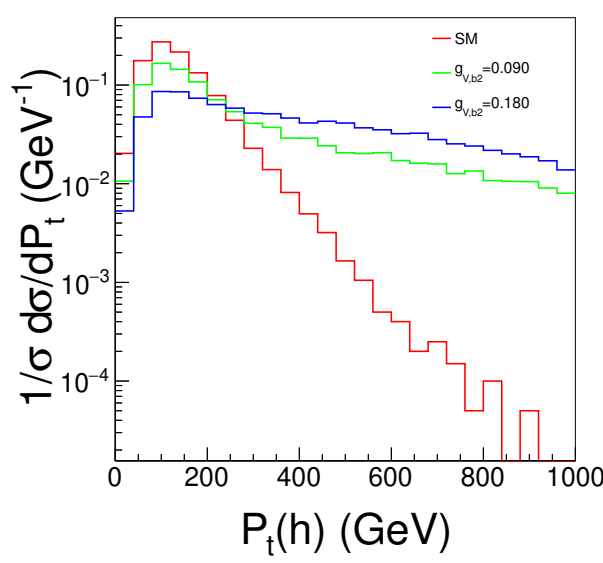

(a)

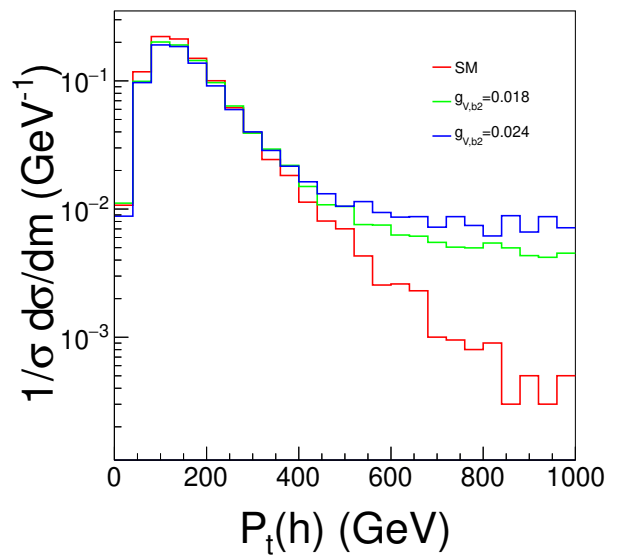

(c)

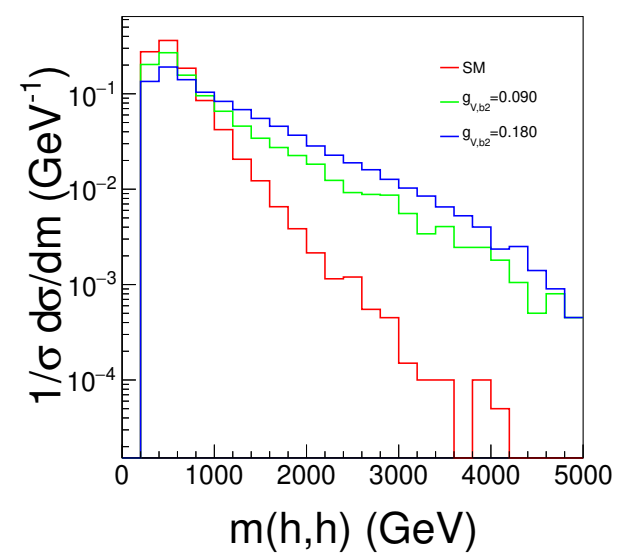

(b)

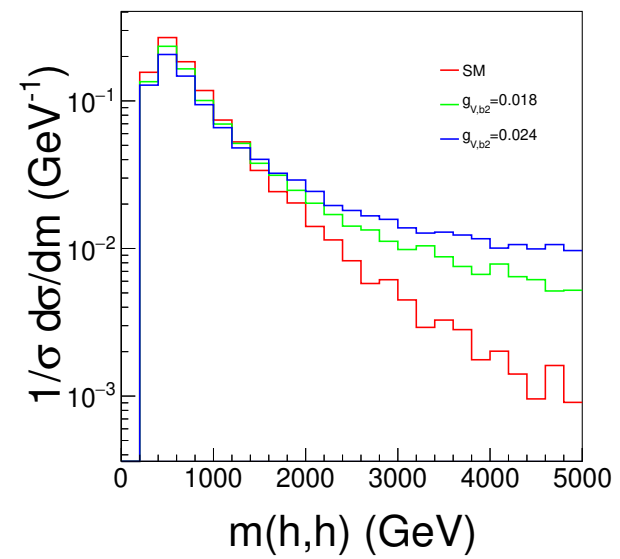

(d)

Figure 4. Distributions of (a) the $P_{t}$ of the leading Higgs and (b) the invariant mass of the Higgs pair at the $14 \mathrm{TeV}$ LHC, with $g_{V, b 2}=0.09$ and $g_{V, b 2}=0.18$. The corresponding distributions for a $100 \mathrm{TeV}$ collider are shown in (c) and (d), with $g_{V, b 2}=0.018$ and $g_{V, b 2}=0.024$.

this parameter value, the total signal cross section $(\sigma \times \mathcal{L})$ is enhanced by a factor of 4 over the SM value. As figure 4a demonstrates, most of enhancement occurs in the boosted region. After b-tagging and $\mathrm{VBF}$ cuts have been applied, there are more than 600 signal events left that can be observed. In table 10 we present the resulting numbers for the three classes of events. In this scenario, the signal accounts for $25 \%$ and $35 \%$ of the total events in the $2 \mathrm{BH}$ and $1 \mathrm{BH}$ classes, respectively. Compared with the results given in table 4 , the increase in signal events in the $2 \mathrm{BH}(1 \mathrm{BH})$ case amounts to a factor $35(10)$, respectively.

When the BDT method is applied, this result is further improved, as shown in figure 5 and table 11. By comparing this with the results of table 4, we conclude that isolating the boosted-Higgs region significantly enhances the discovery potential of this process, both for the $2 \mathrm{BH}$ and $1 \mathrm{BH}$ cases.

By contrast, in the $0 \mathrm{BH}$ case the signal significance is too low for the chosen collider parameters and benchmark values of $g_{V, b 2}$. 


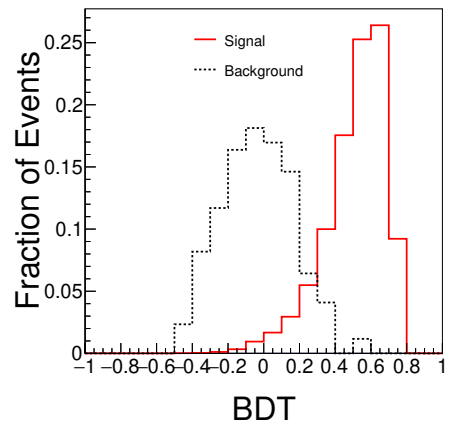

(a) $2 \mathrm{BH}$.

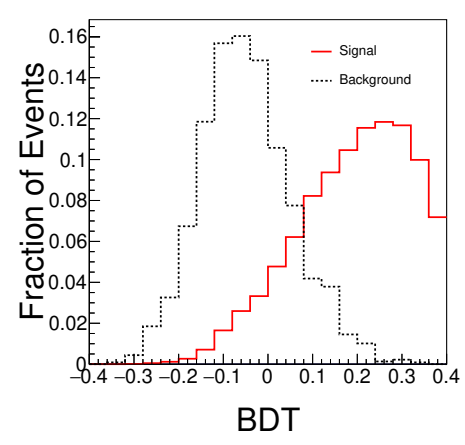

(b) $1 \mathrm{BH}$.

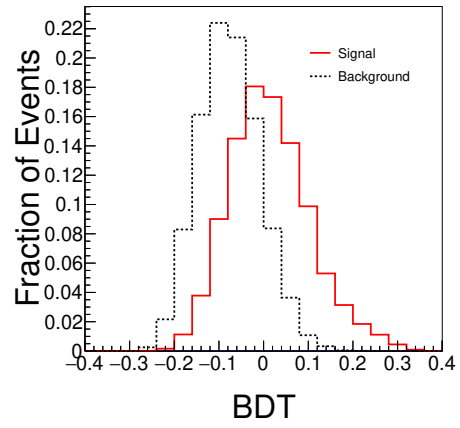

(c) $0 \mathrm{BH}$.

Figure 5. The response of the discriminants to the $g_{V, b 2}$ signal and background at $14 \mathrm{TeV}$ LHC with (a) $2 \mathrm{BH}$, (b) $1 \mathrm{BH}$, and (c) $0 \mathrm{BH}$.

\begin{tabular}{|c|ccc|}
\hline Process & $\sigma \times \mathcal{L}$ & $n_{b}=4$ & $\mathrm{VBF}$ \\
\hline$g_{V, b 2}=0.18$ signal & 4243 & 1019 & 617 \\
\hline$p p \rightarrow 4 b 2 j$ & $2.28 \times 10^{8}$ & $5.47 \times 10^{7}$ & $1.86 \times 10^{7}$ \\
$p p \rightarrow 2 b 4 j$ & $2.38 \times 10^{10}$ & $1.14 \times 10^{4}$ & $3.85 \times 10^{4}$ \\
$p p \rightarrow t \bar{t} \rightarrow 2 b 4 j$ & $7.89 \times 10^{8}$ & 387 & 58 \\
\hline
\end{tabular}

Table 9. The cut efficiencies of b-tagging and VBF at $14 \mathrm{TeV}$ LHC are demonstrated. Here, the total integrated luminosity is assumed to be $\mathcal{L}=3 \mathrm{ab}^{-1}$. b-tagging efficiency is $\epsilon_{b}=0.7$, and mis-tagging rate is $\epsilon_{\text {miss }}=0.001$.

\begin{tabular}{|c|ccc|}
\hline & $\begin{array}{c}\text { 2-boosted Higgs } \\
(2 \mathrm{BH})\end{array}$ & $\begin{array}{c}\text { 1-boosted Higgs } \\
(1 \mathrm{BH})\end{array}$ & $\begin{array}{c}\text { 0-boosted Higgs } \\
(0 \mathrm{BH})\end{array}$ \\
\hline$g_{V, b 2}=0.18$ Signal & 153 & 217 & 247 \\
\hline$p p \rightarrow 4 b 2 j$ & $1.17 \times 10^{5}$ & $1.56 \times 10^{6}$ & $1.69 \times 10^{7}$ \\
$p p \rightarrow 2 b 4 j$ & 28 & 349 & $3.81 \times 10^{4}$ \\
$p p \rightarrow t \bar{t} \rightarrow 2 b 4 j$ & 3 & 13 & 42 \\
\hline
\end{tabular}

Table 10. The numbers of $2 \mathrm{BH}, 1 \mathrm{BH}$ and $0 \mathrm{BH}$ cases at $14 \mathrm{TeV} \mathrm{LHC}$ are tabulated.

\begin{tabular}{|c|ccc|}
\hline & $\begin{array}{c}\text { 2-boosted Higgs } \\
(2 \mathrm{BH})\end{array}$ & $\begin{array}{c}\text { 1-boosted Higgs } \\
(1 \mathrm{BH})\end{array}$ & $\begin{array}{c}\text { 0-boosted Higgs } \\
(0 \mathrm{BH})\end{array}$ \\
\hline$g_{V, b 2}=0.18$ Signal with BDT cut & 54 & 30 & 10 \\
\hline Background with BDT cut & 0 & 0 & 3430 \\
\hline$S / B$ & - & - & $2.99 \times 10^{-3}$ \\
\hline$S / \sqrt{S+B}$ & 7.348 & 5.477 & 0.175 \\
\hline
\end{tabular}

Table 11. The significances of the BDT at $14 \mathrm{TeV}$ collider are demonstrated. 


\subsection{Unitarity limits and discovery reach}

The interactions of Higgs bosons with transverse gauge bosons in the Lagrangian (1.3) involve derivative couplings, and therefore are enhanced over the SM interactions for high values of the four-momenta. This is clearly visible in figure 4 , where the contribution of the new interactions dominates for sufficiently large values of the transverse momentum $P_{t}$ or the Higgs-pair invariant mass $m(h, h)$.

If this behavior is naively extrapolated, the computed amplitudes will violate unitarity constraints. In ref. [4], we have derived a generic unitarity bound for the $p p \rightarrow h h j j$ process, which relates the value of $g_{V, b 2}$ to a UV cutoff $\Lambda_{\mathrm{UV}}$.

$$
\frac{\Lambda_{\mathrm{UV}}^{4}}{2^{9} \pi^{2} v^{4}}\left|g_{V, b 2}\right|^{2} \leq \frac{1}{4}
$$

For energy-momentum values beyond $\Lambda_{\mathrm{UV}}$, the EFT expansion breaks down. We expect higher-order contributions and, eventually, a new structure of underlying interactions to dampen the rise of the amplitudes, e.g., a resonance. Conversely, for the EFT description to remain useful, the value of $g_{V, b 2}$ must be such that the cutoff implied by (3.5) is outside the accessible kinematic range, or we would have to apply a cutoff or form factor on the calculated distributions.

In figure 6 , we display the sensitivity on $\Lambda_{\mathrm{UV}}$ by using the $6 \mathrm{a} 2 \mathrm{BH}$ case and $6 \mathrm{~b} 1 \mathrm{BH}$ case at the $14 \mathrm{TeV}$ LHC, respectively. To this end, we determine the maximally allowed $g_{V, b 2}$ value as a function of $\Lambda_{\mathrm{UV}}$ using (3.5). The y-axis indicates the number of signal events that result for corresponding values of the cutoff $\Lambda_{\mathrm{UV}}$ and the maximal $g_{V, b 2}$, respectively. The dashed blue line, for each plot, marks the $5 \sigma$ discovery threshold as it follows from the signal and background event rates derived above.

The crossing points of the signal curves and the discovery thresholds in figure 6 determine the sensitivity of the analysis to heavy new physics, assuming that the bound (3.5) for $g_{V, b 2}$ is saturated. We conclude that the effect of a heavy resonance, for instance, can be accessible up to $4.4 \mathrm{TeV}$ in the $2 \mathrm{BH}$ case and up to $3.6 \mathrm{TeV}$ in the $1 \mathrm{BH}$ case. The cleaner environment of the $2 \mathrm{BH}$ case is clearly preferred. By contrast, the discovery reach of the $0 \mathrm{BH}$ case is limited to about $2.4 \mathrm{TeV}$.

We can apply the analogous analysis to a $100 \mathrm{TeV}$ hadron collider. Based on the results obtained for the LHC with $\sqrt{s}=14 \mathrm{TeV}$ where the $2 \mathrm{BH}$ case has been most useful, below we only present the results for this analysis which requires two highly boosted Higgs bosons.

As shown above, at a $100 \mathrm{TeV}$ machine the signal can be discovered already in the SM case where no enhancement is present. Therefore, we define the observability of new physics in terms of the deviation in rate, $N_{2 \mathrm{BH}}-N_{2 \mathrm{BH}}^{\mathrm{SM}}$, where $N_{2 \mathrm{BH}}^{\mathrm{SM}}$ is the number of events in the $2 \mathrm{BH}$ category in the SM case.

By using the BDT analysis as before, we derive the $5 \sigma$ excess bound for new physics at the $100 \mathrm{TeV}$ collider, as marked in figure 7 by the blue dashed line. We read off this figure that this collider can yield a meaningful constraint on $g_{V, b 2}$ at a value which corresponds to a sensitivity in the new-physics scale of $\Lambda_{\mathrm{UV}}=27 \mathrm{TeV}$. 


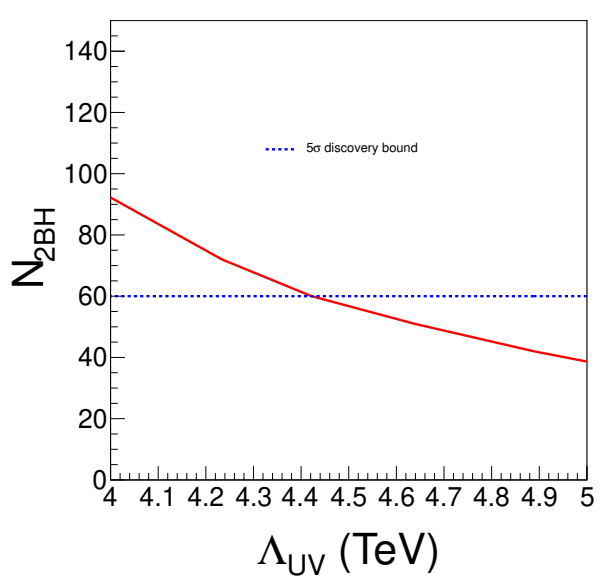

(a)

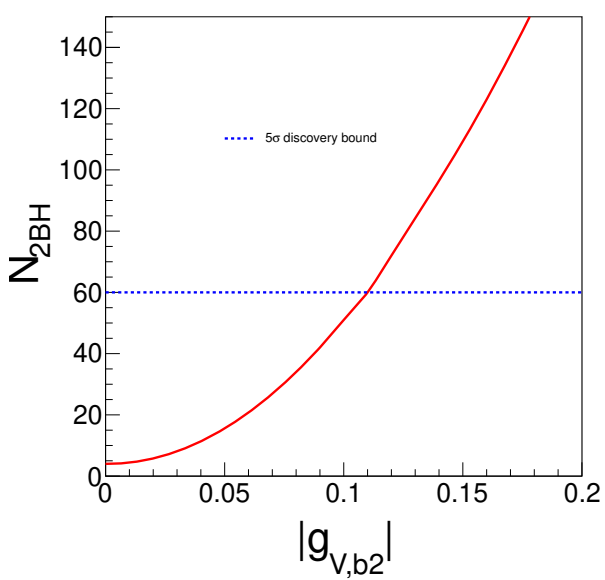

(c)

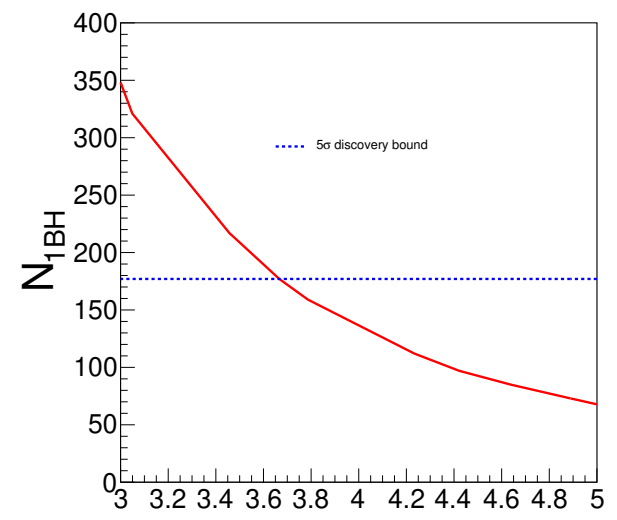

$\Lambda_{\mathrm{UV}}(\mathrm{TeV})$

(b)

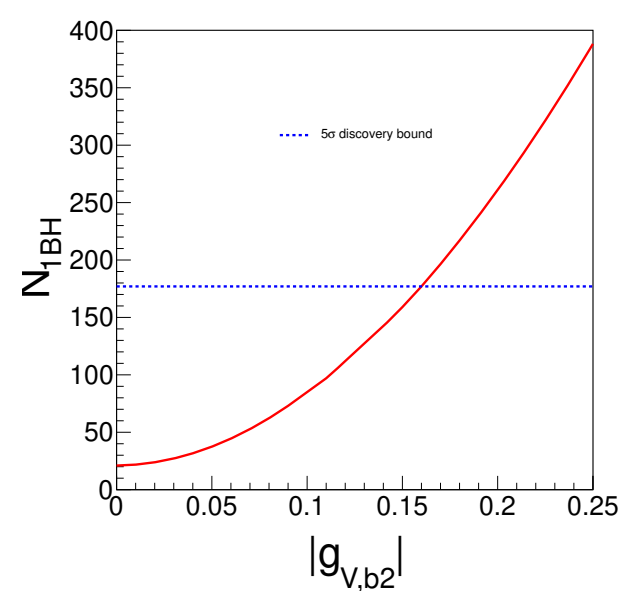

(d)

Figure 6. The $5 \sigma$ discovery constraints on new physics cutoff at $14 \mathrm{TeV}$ LHC are demonstrated. (a) is the constraint obtained from $2 \mathrm{BH}$ case, and (b) is the constraint obtained from $1 \mathrm{BH}$ case. (c) and (d) are the corresponding constraints on the $g_{V, b 2}$ in the $2 \mathrm{BH}$ and $1 \mathrm{BH}$ case, respectively.

\section{Two-parameter bounds}

So far, we have considered only the dependence of the Higgs-pair VBF process on the couplings $g_{V, b 2}$ to the transverse polarization of vector bosons, which dominate the distribution in the highly boosted region. In this section, we complement the discussion by taking into account also the $g_{V, a 2}$ couplings which describe the interaction of a Higgs pair with longitudinally polarized vector bosons. This interaction exists in the SM but can receive a correction if dimension-6 operators are included.

In order to express the cross sections of $p p \rightarrow h h j j$ in terms of the parameters given in eq. (1.1), we impose some simplifying assumptions on the phenomenological information from expected data on single-Higgs processes. For instance, the $W W h$ vertex should be strongly constrained by data from the Higgs decay to $W W$ as well as from VBF singleHiggs production. As stated before, we ignore the universal ambiguity in the Higgs-coupling 


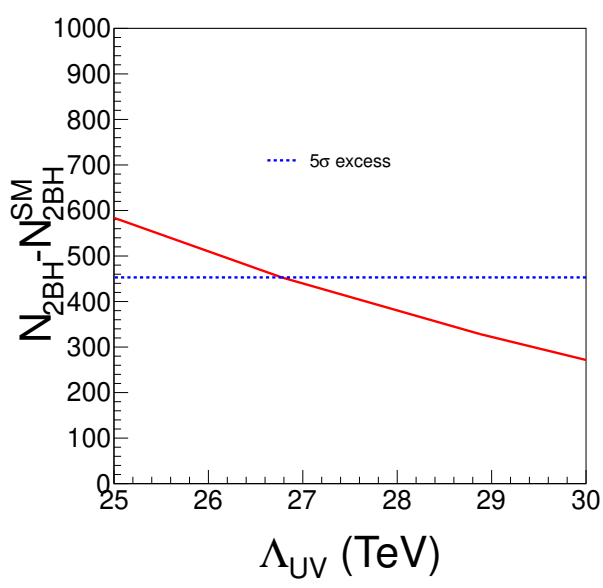

(a)

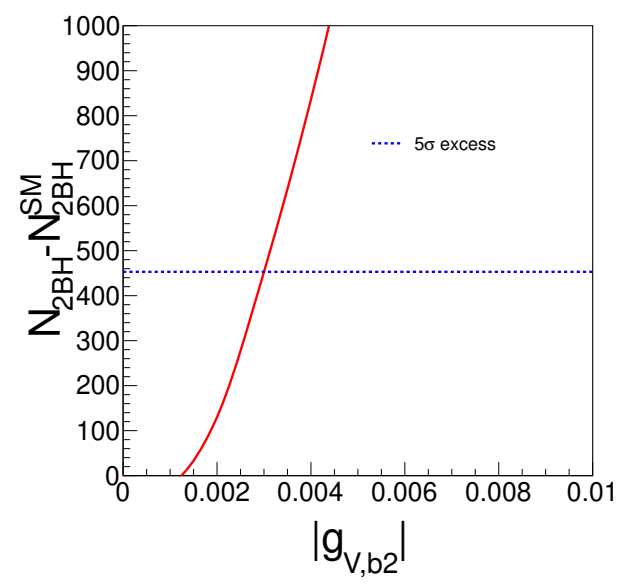

(b)

Figure 7. The $5 \sigma$ excess constraint obtained from $2 \mathrm{BH}$ events on new physics cutoff at $100 \mathrm{TeV}$ hadron collider is demonstrated in (a), while (b) is the corresponding $g_{V, b 2}$.

\begin{tabular}{|c|c|c|c|}
\hline & $\sigma_{a_{2}}^{0}(\mathrm{fb})$ & $\sigma_{a_{2}}^{1}(\mathrm{fb})$ & $\sigma_{a_{2}}^{2}(\mathrm{fb})$ \\
\hline $14 \mathrm{TeV}$ & 17.71 & -29.33 & 12.68 \\
\hline $27 \mathrm{TeV}$ & 88.3 & -152.2 & 68.1 \\
\hline $100 \mathrm{TeV}$ & 1601.2 & -2963.8 & 1401 \\
\hline
\end{tabular}

Table 12. Coefficients $\sigma_{a_{2}}^{0}, \sigma_{a_{2}}^{1}$, and $\sigma_{a_{2}}^{2}$ in the expression (4.1) for $p p \rightarrow h h j j$ at three different collider energies.

determination due to undetected Higgs decays at the LHC, which can be lifted by modelindependent measurements at Higgs factories such as the CEPC, the ILC, or the CLIC collider. Accordingly, we fix $g_{W, a 1}$ and $g_{W, b 1}$, the single-Higgs couplings to longitudinal and transverse $W$ polarizations, to their SM values. As a further simplification, we assume the custodial-symmetry relations $g_{W, a i}=g_{Z, a i}$ and $g_{W, b i}=g_{Z, b i}(i=1,2)$ whenever contributions of $\mathrm{Z}$ bosons are considered.

Since the amplitudes are at most linear in the parameters, we can parameterize the cross section of $p p \rightarrow h h j j$ in terms of $g_{V, a 2}$ as given below

$$
\sigma(p p \rightarrow h h j j)=\sigma_{a_{2}}^{0}+\sigma_{a_{2}}^{1} g_{V, a 2}+\sigma_{a_{2}}^{2} g_{V, a 2}^{2} .
$$

We compute the coefficients $\sigma_{a_{2}}^{0}, \sigma_{a_{2}}^{1}$, and $\sigma_{a_{2}}^{2}$ numerically using Monte-Carlo methods, evaluating the total cross section for a sufficient number of different coupling values. The results are given in table 12 .

Analogously, we can parameterize the cross section as a function on $g_{V, b 2}$ as follows

$$
\sigma(p p \rightarrow h h j j)=\sigma_{b_{2}}^{0}+\sigma_{b_{2}}^{1} g_{V, b 2}+\sigma_{b_{2}}^{2} g_{V, b 2}^{2},
$$

The numerical results for $\sigma_{b_{2}}^{0}, \sigma_{b_{2}}^{1}$, and $\sigma_{b_{2}}^{2}$ are given in table 13 . 


\begin{tabular}{|c|c|c|c|}
\hline & $\sigma_{b_{2}}^{0}(\mathrm{fb})$ & $\sigma_{b_{2}}^{1}(\mathrm{fb})$ & $\sigma_{b_{2}}^{2}(\mathrm{fb})$ \\
\hline $14 \mathrm{TeV}$ & 1.06 & 1.52 & 106.8 \\
\hline $27 \mathrm{TeV}$ & 4.2 & 6.97 & 1135.2 \\
\hline $100 \mathrm{TeV}$ & 38.4 & 83.08 & 54070 \\
\hline
\end{tabular}

Table 13. Coefficients $\sigma_{b_{2}}^{0}, \sigma_{b_{2}}^{1}$, and $\sigma_{b_{2}}^{2}$ in the expression (4.2) for $p p \rightarrow h h j j$ at three different collider energies.

\begin{tabular}{|c|ccc|}
\hline & $14 \mathrm{TeV}\left(3 \mathrm{ab}^{-1}\right)$ & $27 \mathrm{TeV}\left(3 \mathrm{ab}^{-1}\right)$ & $100 \mathrm{TeV}\left(30 \mathrm{ab}^{-1}\right)$ \\
\hline$\delta g_{V, a 2}$ & $(-0.31,0.39)$ & $(-0.11,0.13)$ & $(-0.013,0.047)$ \\
\hline$g_{V, b 2}$ & $(-0.10,0.11)$ & $(-0.03,0.02)$ & $(-0.003,0.003)$ \\
\hline
\end{tabular}

Table 14. $5 \sigma$ discovery (excess) bounds of $g_{V, a 2}$ and $g_{V, b 2}$ at a $14 \mathrm{TeV}, 27 \mathrm{TeV}$ and $100 \mathrm{TeV}$ hadron collider, assuming the respective total integrated luminosity in brackets.

When both $g_{V, b 2}$ and $g_{V, a 2}$ are turned on, we have to include a mixed coefficient proportional to $g_{V, b 2} g_{V, a 2}$. The corresponding results can be found in [4]; the values are $1.7 \mathrm{fb}$ for $14 \mathrm{TeV}, 9.6 \mathrm{fb}$ for $27 \mathrm{TeV}$, and $95 \mathrm{fb}$ for $100 \mathrm{TeV}$, respectively.

The numerical results given in table 12 and table 13 have been obtained with Madgraph5 and Whizard by independent calculations, with very good numerical agreement. It should be pointed out that the results in table 12 clearly reflect the strong gauge cancellation which occurs between individual terms of (4.1) in the SM limit. Some of the coefficients $\sigma_{a_{2}}^{0}, \sigma_{a_{2}}^{1}$, and $\sigma_{a_{2}}^{2}$ are one order of magnitude larger than that of the cross section in the SM, as given as $\sigma_{b_{2}}^{0}$ in table 13. Using this parameterization and applying the results of our study, we derive the parameter ranges tabulated in table 14. Outside the given limits, the deviation from the SM can be detectable as a $5 \sigma$ discovery.

We show the projected bounds on $g_{V, a 2}$ and $g_{V, b 2}$ in figure 8 . We have compared our results for $g_{V, a 2}$ with those given in ref. [29] and found good agreement. Regarding the unitarity bounds shown in the plots, the bounds on $g_{V, b 2}$ correspond to eq. (3.5), while for $g_{V, a 2}$ we make use of the result [4]

$$
\frac{\Lambda_{\mathrm{UV}}^{4}}{2^{9} \pi^{2} v^{4}}\left|g_{V, a 2}-g_{V, a 1}^{2}\right|^{2} \leq \frac{1}{4}
$$

It is interesting to explore whether it is possible to disentangle the effects of the operator $h^{2} W_{\mu \nu}^{a} W^{a, \mu \nu}$ from those of the operator $h^{2} W_{\mu}^{a} W^{a, \mu}$. As mentioned in our previous work [4], the azimuthal angle between two forward jets can be a useful observable for this purpose.

In figure 9, we display the azimuthal angle distributions for the $14 \mathrm{TeV}, 27 \mathrm{TeV}$ and $100 \mathrm{TeV}$ cases. We take into account only events in category $2 \mathrm{BH}$ and apply no further cuts beyond the VBF cuts. The relative azimuthal angle is defined as $\Delta \phi=\left|\phi\left(j_{1}\right)-\phi\left(j_{2}\right)\right|$, where $\phi\left(j_{1}\right)$ denotes the azimuthal angle of the leading forward jet, and $\phi\left(j_{2}\right)$ denotes that of the second leading forward jet. 


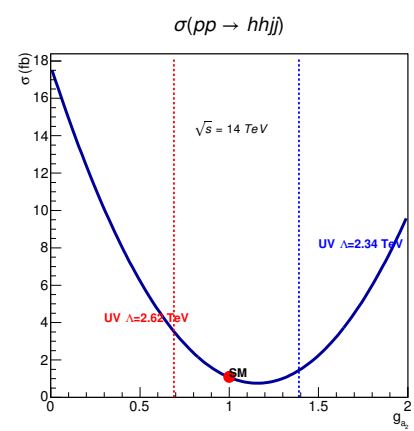

(a) $14 \mathrm{TeV}$.

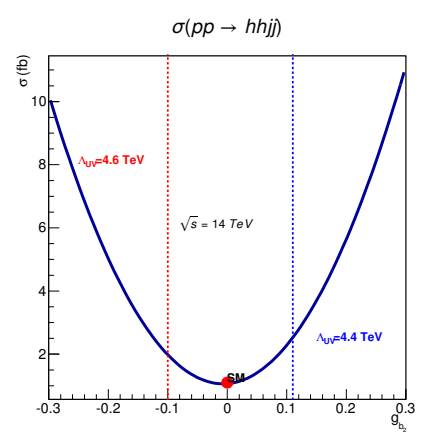

(d) $14 \mathrm{TeV}$.

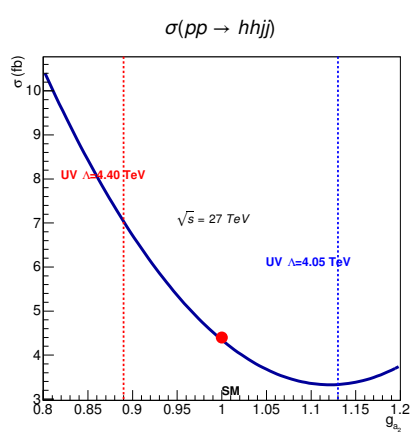

(b) $27 \mathrm{TeV}$.

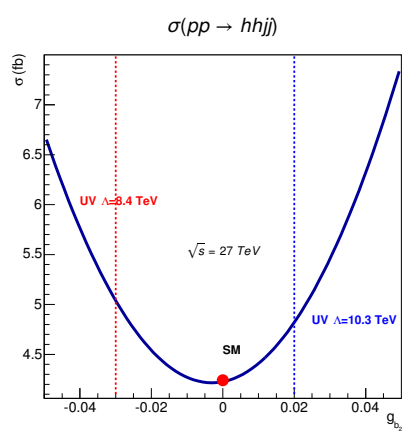

(e) $27 \mathrm{TeV}$.

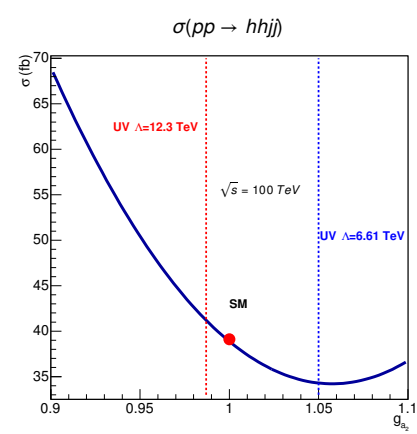

(c) $100 \mathrm{TeV}$.

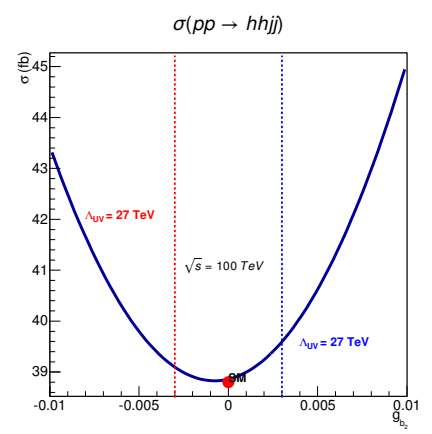

(f) $100 \mathrm{TeV}$.

Figure 8. Total cross section after VBF cuts for the process $p p \rightarrow h h j j$ as a function of the $W W h h$ couplings $g_{V, a 2}$ (upper row) and $g_{V, b 2}$ (lower row), for three different collider energies. The vertical lines are unitarity bounds, which are derived from eq. (4.3) and eq. (3.5).

We note the main features of the distributions given in figure 9:

- In the SM, the azimuthal-angle distribution of the SM is flat due to the dominance of longitudinal polarized vector bosons in the process $p p \rightarrow h h j j$, as shown by the red curve in each of plot. Similarly, the distribution is also flat for the case where the term $h^{2} W_{\mu}^{a} W^{a, \mu}$ is turned on, as shown by the green curve in each of plot where both the contrbutions of the SM and new physics have been included. Although new physics represented by the operator $h^{2} W_{\mu}^{a} W^{a, \mu}$ enhances the total cross section, the azimuthal angle distribution remains similar to that of the SM.

- If the operator $h^{2} W_{\mu \nu}^{a} W^{a, \mu \nu}$ is turned on, the Higgs pair being coupled to transversely polarized vector bosons leads to distributions that significantly differ from that of the SM. As the blue curves indicate, this type of new interaction causes more events with back-to-back outgoing jets.

- At the $100 \mathrm{TeV}$ collider, the difference in shape of the angular distribution is much less pronounced. In fact, the chosen benchmark values of the operator coefficients cause just a small disturbance of the SM signal in this plot, which is still detectable due to the much larger event rate for the high energy and high luminosity of the 


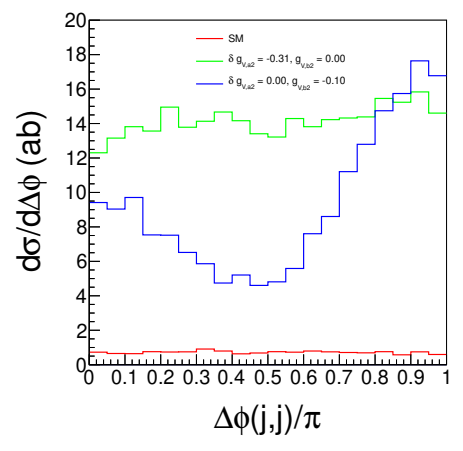

(a) $14 \mathrm{TeV}$.

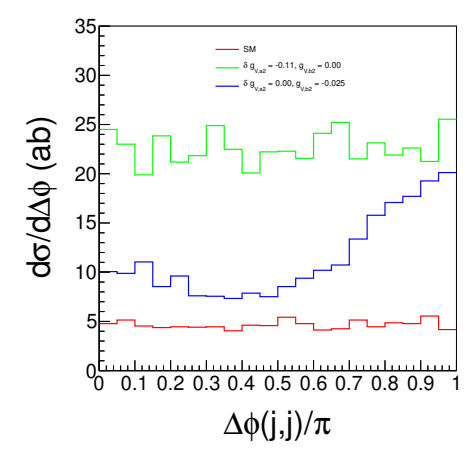

(b) $27 \mathrm{TeV}$.

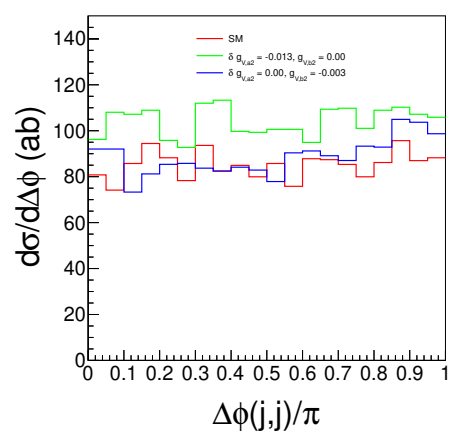

(c) $100 \mathrm{TeV}$.

Figure 9. The relative azimuthal angle distributions of two forward jets for $14 \mathrm{TeV}, 27 \mathrm{TeV}$, and $100 \mathrm{TeV}$.
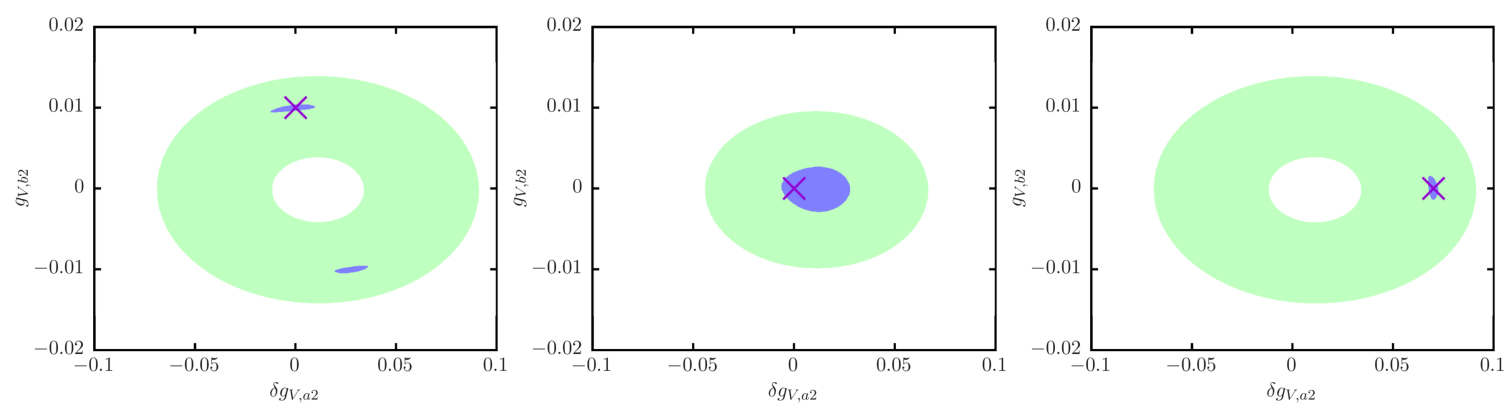

Figure 10. The fit results with inclusive cross section (green) and differential cross section (blue) with three different assumptions on the central values: $\left(\delta g_{V, a 2}^{\text {true }}, g_{V, b 2}^{\text {true }}\right)=(0,0),(0.07,0),(0,0.01)$ with $\sqrt{s}=100 \mathrm{TeV}$ and an integrated luminosity $30 \mathrm{ab}^{-1}$.

machine. To distinguish the two kinds of contributions, a more detailed analysis of this and other distributions becomes necessary.

To fully utilise the differential information, we perform a $\chi^{2}$-fit on the distribution of $\Delta \phi(j, j)$, using the $2 \mathrm{BH}$ events after the BDT cuts with the collision energy $\sqrt{s}=100 \mathrm{TeV}$ and integrated luminosity $30 \mathrm{ab}^{-1}$. In figure 10, we show the $2 \sigma$ allowed region obtained with differential information (blue) or just from the total cross section (green). For the leftmost plot, we assume the SM for the true values of the coefficients. It is evident that the information from the differential distribution significantly improves the precisions on both $\delta g_{V, a 2}$ and $g_{V, b 2}$. The middle plot assumes $\left(\delta g_{V, a 2}^{\text {true }}, g_{V, b 2}^{\text {true }}\right)=(0.07,0)$ for the true value, i.e., only $g_{V, a 2}$ receives a new-physics contribution. The inclusive cross section confines the region allowed by a measurement to a ring, due to the quadratic dependence on both $\delta g_{V, a 2}$ and $g_{V, b 2}$, while the differential distribution singles out a small area around the point $\left(\delta g_{V, a 2}, g_{V, b 2}\right)=(0.07,0)$. Analogously, for the right plot we assume the true values $\left(\delta g_{V, a 2}^{\text {true }}, g_{V, b 2}^{\text {true }}\right)=(0,0.07)$. Again, the differential distribution selects a small region, but in this case there is a two-fold sign ambiguity left for $g_{V, b 2}$. This reflects the fact that the effects of $g_{V, b 2}$ is dominated by the squared term, and thus the sign of $g_{V, b 2}$ cannot be determined. 
Finally, using the relations given in table 8, we can map this contour onto the plane spanned by $c_{W}$ and $c_{H W}$. The assumption of a linearly realized symmetry and truncation at the dimension- 6 order enforces the constraint $\delta g_{V, a 2} \sim 6 g_{V, b 2}$, if both couplings depend only on a single parameter $c_{H W}$. The two-parameter analysis that we describe in this paper, would allow us to search for the relation of $c_{W}$ and $c_{H W}$, which would point to the presence of contributions that do not follow the simple power-counting assumption underlying the dimension-six truncation of the SILH basis. Currently, the experimental data constraints on the parameter set are rather weak, to the level of $c_{V} \sim O\left(10^{3}\right)$ [83]. In this study, we conclude that we can reach a sensitivity of up to $c_{V} \sim O(10)$ at future colliders.

\section{Discussions and conclusions}

We have studied double-Higgs production in vector-boson at a proton-proton collider, $p p \rightarrow$ $h h j j \rightarrow 4 b 2 j$. We compare the $14 \mathrm{TeV}$ LHC with future high-energy and high-luminosity colliders of $27 \mathrm{TeV}$ and $100 \mathrm{TeV}$. The analysis of this process benefits greatly from identifying highly-boosted Higgs bosons. To this end, we use the mass-drop method to analyse the jet substructure, and we optimize the significance by the boosted decision-tree method. Our results show that the $2 \mathrm{BH}$ case where two highly-boosted Higgs are tagged can provide the cleanest experimental environment to discover new physics in the VBF signal.

At the LHC, the number of $2 \mathrm{BH}$ events is too small to discover this channel if the SM is valid without new contributions. Conversely, at a $100 \mathrm{TeV}$ collider with a high luminosity of the order of $30 \mathrm{ab}^{-1}$, the number of $2 \mathrm{BH}$ events is large enough to discover the SM signal.

To study the effects of new physics, we use a phenomenological effective Lagrangian (1.3). We explore the effect of the interactions of type $h^{2} V_{\mu}^{a} V^{a, \mu}$ and $h^{2} V_{\mu \nu}^{a} V^{a, \mu \nu}$ and extract bounds for the associated coefficients $g_{V, a 2}$ and $g_{V, b 2}$. Our results demonstrate that new-physics scales up to $4.4 \mathrm{TeV}$ at the $14 \mathrm{TeV} \mathrm{LHC}$, and $27 \mathrm{TeV}$ at a $100 \mathrm{TeV}$ hadron collider, are within reach of discovery.

Figure 11 collects the projected bounds on $g_{V, a 2}$ and $g_{V, b 2}$ that we have determined in this work, cf. also table 14 . The bounds that can be obtained at $27 \mathrm{TeV}$, are one order of magnitude stronger than for the $14 \mathrm{TeV}$ LHC. The $100 \mathrm{TeV}$ machine can further constrain these parameters by another order of magnitude. At both the $27 \mathrm{TeV}$ and $100 \mathrm{TeV}$ machines, this offers a significant indirect sensitivity to new physics in this sector.

We have neglected the background processes $g g \rightarrow h h j j$ in our analysis, which has been taken into account in [28]. When we compute the cross section of this process in the SM by using Madgraph5.0@NLO, we find that after VBF cuts, its cross section is $0.022 \mathrm{fb}$ when $\sqrt{s}=14 \mathrm{TeV}$, which is only $38 \%$ of that of the signal processes, and it is negligibly when compared with the huge QCD background process $p p \rightarrow 4 b j j$. Even when we include such a background process in our analysis, our results does not change. When there are new physics operators like $g_{t t h h} t \bar{t} h^{2}$ and $\lambda_{3} h^{3}$ which might contribute to the processes $g g \rightarrow h h j j$ significantly, then the measurement of $g_{V, a 2}$ and $g_{V, b 2}$ might be significantly affected by the uncertainties of these couplings. In reality, the couplings like $g_{t t h h}$ and $\lambda_{3}$ should firstly be measured from those processes like $p p \rightarrow t t h h$ and $g g \rightarrow h h$, which 


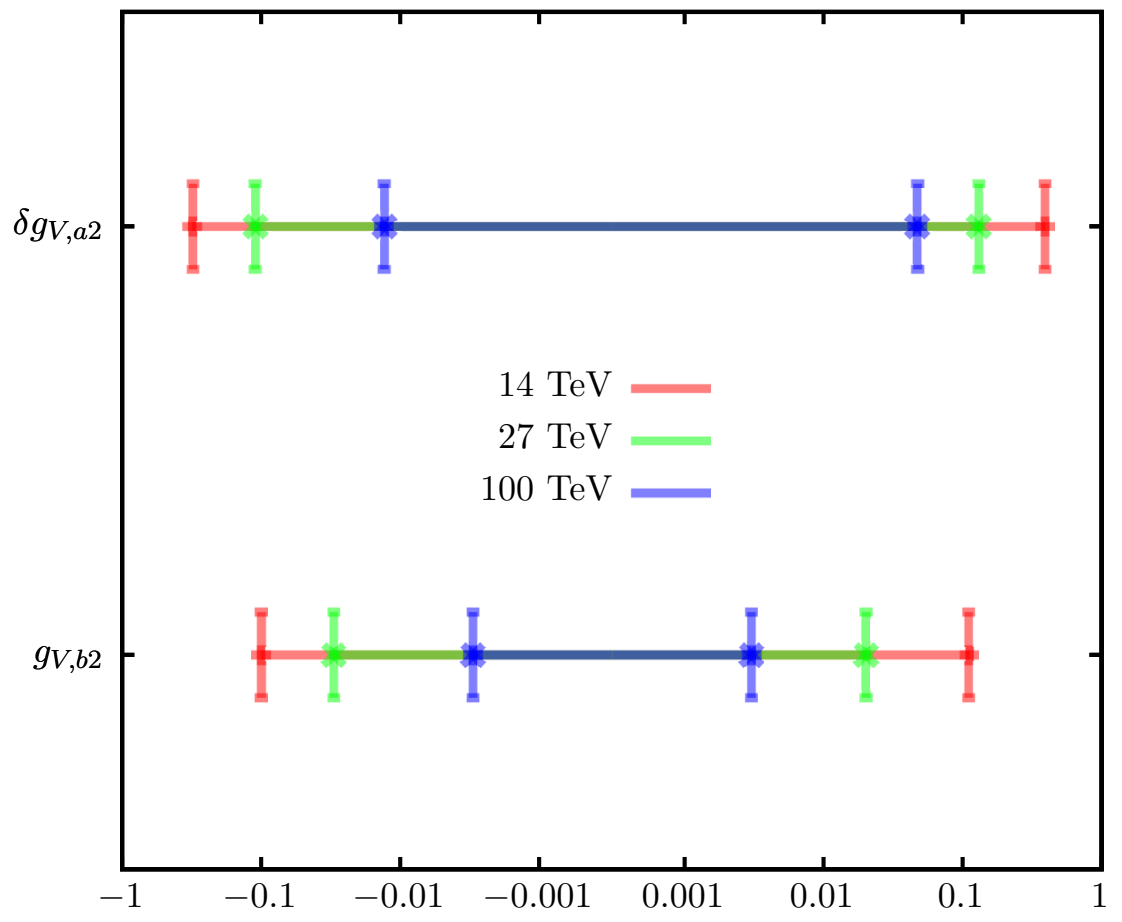

Figure 11. The summarized bounds of $g_{V, a 2}$ and $g_{V, b 2}$ at $14 \mathrm{TeV}, 27 \mathrm{TeV}$ and $100 \mathrm{TeV}$ hadron collider, which is the same as table 14 .

have much larger cross sections and are more sensitive to these couplings when the same collision energy and luminosity are assumed. In this case, a central jet veto can help to suppress events from such a background process, as observed in [26, 28]. In experimental analysis, new physics operators can be taken into account to make a global fit by including all accessible experimental processes. Then the bounds of $g_{V, a 2}$ and $g_{V, b 2}$ can be modified when different assumptions are made. Obviously, in this work, our analysis has assumed that experimental measurements can constrained these new couplings (say $g_{t t h h}$ and $\lambda_{3}$ etc.) to a certain level, i.e. a level where the contribution of $g g \rightarrow h h j j$ can be safely neglected. In other words, we have neglected the potential effects of other operators from new physics. The bounds of $g_{V, a 2}$ and $g_{V, b 2}$ can be significantly affected when more other operators are introduced and the correlations among the bounds of these parameters deserve our future studies.

Considering the large background events from the SM processes, the rejection of background events is very crucial in our analysis. In our analysis, it is observed that the number of SM background events $p p \rightarrow 2 b 4 j$ is several order larger than that of the signal events. Therefore, a workable strategy is to firstly use b tagging (with a smaller cone size, say $R=0.4$ or smaller) to reject the SM background events to a manageable and computable level. After that, the jet substructure analysis can be applied to massive jets reclustered with a larger cone parameter say $R=1.2$. Therefore we first impose 4 tagged b jets to suppress the total number of events (especially events of $264 j$ ), which can suppress the background events by a factor $10^{6}$. While the substructure criteria and a cut on the mass 
of tagged Higgs jets (imposed as $\left|m_{h}-125\right|<25 \mathrm{GeV}$ ) can only suppress the background events from $4 b 2 j$ by a factor $10^{3}$. Obviously, the jet substructure criteria can not suppress the background events as sufficiently as 4 b-taggings. Usually a b-tagging is performed after the substructure analysis as demonstrated in the BDRS paper, but in a realistic experimental analysis, the order of substructure analysis and b-taggings is exchangeable. B-tagging can be implemented at the trigger level and could be prior to the jet substructure analysis (jet substructure analysis depends upon jet algorithms and jet parameters, which can be done at the off-line level (or called third level)). Considering the fact that the background is so huge in the SM, obviously the usual single b-tagging in the BDRS method is not sufficient to suppress the SM background events, especially $2 b 4 j$. Therefore it is reasonable to require two tagged b-jets inside each of highly boosted Higgs jets of every event.

In this work, we have resorted to the BDT method to optimize the signal and background discrimination. And it is observed that the BDT method indeed is very crucial and important to improve the sensitivity. For example, when compared with the cut based method, say in the $2 \mathrm{BH}$ case with a collision energy $14 \mathrm{TeV}$ and a $3 / a b$ dataset, a single cut based method (say $m_{H}-125 \mid<25 \mathrm{GeV}$ ) can only yield a significance 0.592 . In contrast, the significance of BDT including all observables can yield a significance 7.348 as given in table 11, which is much better.

Our study does not account for underlying-event or pile-up effects. These produce additional soft radiation and may render the extraction of a hard-process signal more difficult. In fact, the mass-drop method selects two-pronged jets in the final state. This reduces the impact of QCD jets from underlying events or pile-up, which are one-pronged. We also use filtering to remove soft radiation after reconstructing two sub-jets in the final state, which is helpful to reject jets from extra radiation produced by the parton shower. Recent studies indicate that modern pile-up mitigation techniques [84] can minimize the pile-up contamination efficiently for the $4 \mathrm{~b}$ final state [57]. A detailed pile-up analysis can be done but is beyond the scope of the current paper.

For a further improvement of our result, we may consider color-flow properties [85] as a tool to further discriminate $h \rightarrow b \bar{b}$ decays from $\mathrm{b}$ jets in the QCD background. Color-connection information can be quantified by observables such as the pull vector [86]. In a recent study of double Higgs production at LHC [87], it was argued that while the color flow is very different between the double-Higgs signal and the QCD background, this information may be diluted after applying kinematics cuts. The authors of [87] proposed to use jet-image and a Deep Neutral Network analysis methods to discriminate the signal from background, rather than constructing the pull vector. We defer these refinements of our study to future work.

In summary, the analysis of highly boosted Higgs-boson pairs in vector-boson fusion is promising and can be used to significantly improve our knowledge about Higgs-sector interactions. In particular, the method should become important for a future high-energy proton collider where the sensitivity is sufficient to extract a signal down to the SM rate. 


\section{Acknowledgments}

W.K. is supported by the Deutsche Forschungsgemeinschaft (DFG, German Research Foundation) under grant 396021762 - TRR 257. S. Sun is supported by MIUR in Italy under Contract (No. PRIN 2015P5SBHT) and ERC Ideas Advanced Grant (No. 267985) "DaMeSyFla"; Q.S. Yan is supported by the Natural Science Foundation of China under the grant NO. 11475180 and NO. 11875260. X.R. Zhao is supported by the "Fonds Spécial de Recherche" (FSR) of the UCLouvain, and by the Italian Ministry of Research (MUR) under grant PRIN 20172LNEEZ.

Open Access. This article is distributed under the terms of the Creative Commons Attribution License (CC-BY 4.0), which permits any use, distribution and reproduction in any medium, provided the original author(s) and source are credited.

\section{References}

[1] ATLAS collaboration, Observation of a new particle in the search for the Standard Model Higgs boson with the ATLAS detector at the LHC, Phys. Lett. B 716 (2012) 1 [arXiv: 1207.7214] [INSPIRE].

[2] CMS collaboration, Observation of a New Boson at a Mass of $125 \mathrm{GeV}$ with the CMS Experiment at the LHC, Phys. Lett. B 716 (2012) 30 [arXiv:1207.7235] [InSPIRE].

[3] D.R.T. Jones and S.T. Petcov, Heavy Higgs Bosons at LEP, Phys. Lett. B 84 (1979) 440 [INSPIRE].

[4] W. Kilian, S. Sun, Q.-S. Yan, X. Zhao and Z. Zhao, Multi-Higgs boson production and unitarity in vector-boson fusion at future hadron colliders, Phys. Rev. D 101 (2020) 076012 [arXiv: 1808.05534] [INSPIRE].

[5] Y.-H. Qi, J.-H. Yu and S.-H. Zhu, Effective field theory perspective on next-to-minimal composite Higgs models, Phys. Rev. D 103 (2021) 015013 [arXiv: 1912.13058] [InSPIRE].

[6] P. Agrawal, D. Saha, L.-X. Xu, J.-H. Yu and C.-P. Yuan, Determining the shape of the Higgs potential at future colliders, Phys. Rev. D 101 (2020) 075023 [arXiv:1907.02078] [INSPIRE].

[7] L.-X. Xu, J.-H. Yu and S.-H. Zhu, Holographic Completion of Minimal Neutral Naturalness Model and Deconstruction, arXiv:1905.12796 [INSPIRE].

[8] H.-L. Li, L.-X. Xu, J.-H. Yu and S.-H. Zhu, EFTs meet Higgs Nonlinearity, Compositeness and (Neutral) Naturalness, JHEP 09 (2019) 010 [arXiv: 1904.05359] [INSPIRE].

[9] ATLAS collaboration, Measurement of the Higgs boson coupling properties in the $H \rightarrow Z Z^{*} \rightarrow 4 \ell$ decay channel at $\sqrt{s}=13$ TeV with the ATLAS detector, JHEP 03 (2018) 095 [arXiv: 1712.02304] [INSPIRE].

[10] CMS collaboration, Measurements of properties of the Higgs boson decaying into the four-lepton final state in pp collisions at $\sqrt{s}=13$ TeV, JHEP 11 (2017) 047 [arXiv: 1706.09936] [INSPIRE].

[11] ATLAS collaboration, Measurements of gluon-gluon fusion and vector-boson fusion Higgs

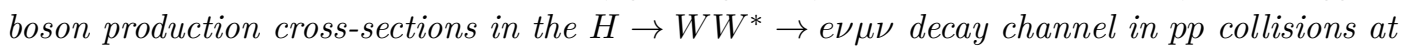
$\sqrt{s}=13 \mathrm{TeV}$ with the ATLAS detector, Phys. Lett. B 789 (2019) 508 [arXiv:1808.09054] [INSPIRE]. 
[12] ATLAS collaboration, Combined measurements of Higgs boson production and decay using up to $80 \mathrm{fb}^{-1}$ of proton-proton collision data at $\sqrt{\mathrm{s}}=13 \mathrm{TeV}$ collected with the ATLAS experiment, Phys. Rev. D 101 (2020) 012002 [arXiv: 1909.02845] [INSPIRE].

[13] ATLAS collaboration, Search for the $H H \rightarrow b \bar{b} b \bar{b}$ process via vector-boson fusion production using proton-proton collisions at $\sqrt{s}=13 \mathrm{TeV}$ with the ATLAS detector, JHEP 07 (2020) 108 [Erratum JHEP 01 (2021) 145] [arXiv: 2001.05178] [INSPIRE].

[14] ATLAS collaboration, Search for pair production of Higgs bosons in the $b \bar{b} b \bar{b}$ final state using proton-proton collisions at $\sqrt{s}=13 \mathrm{TeV}$ with the ATLAS detector, JHEP 01 (2019) 030 [arXiv: 1804.06174] [INSPIRE].

[15] CMS collaboration, Search for nonresonant Higgs boson pair production in the b $\bar{b} b \bar{b}$ final state at $\sqrt{s}=13 \mathrm{TeV}$, JHEP 04 (2019) 112 [arXiv:1810.11854] [INSPIRE].

[16] ATLAS collaboration, Search for Higgs boson pair production in the $\gamma \gamma b \bar{b}$ final state with $13 \mathrm{TeV}$ pp collision data collected by the ATLAS experiment, JHEP 11 (2018) 040 [arXiv: 1807.04873] [INSPIRE].

[17] CMS collaboration, Search for Higgs boson pair production in the $\gamma \gamma b \bar{b}$ final state in $p p$ collisions at $\sqrt{s}=13 \mathrm{TeV}$, Phys. Lett. B 788 (2019) 7 [arXiv:1806.00408] [InSPIRE].

[18] ATLAS collaboration, Search for resonant and non-resonant Higgs boson pair production in the $b \bar{b} \tau^{+} \tau^{-}$decay channel in pp collisions at $\sqrt{s}=13 \mathrm{TeV}$ with the ATLAS detector, Phys. Rev. Lett. 121 (2018) 191801 [Erratum ibid. 122 (2019) 089901] [arXiv:1808.00336] [INSPIRE].

[19] CMS collaboration, Search for Higgs boson pair production in events with two bottom quarks and two tau leptons in proton-proton collisions at $\sqrt{s}=13 \mathrm{TeV}$, Phys. Lett. B $\mathbf{7 7 8}$ (2018) 101 [arXiv: 1707.02909] [INSPIRE].

[20] ATLAS collaboration, Search for Higgs boson pair production in the $b \bar{b} W W^{*}$ decay mode at $\sqrt{s}=13 \mathrm{TeV}$ with the ATLAS detector, JHEP 04 (2019) 092 [arXiv: 1811.04671] [INSPIRE].

[21] CMS collaboration, Search for resonant and nonresonant Higgs boson pair production in the $b \bar{b} \ell \nu \ell \nu$ final state in proton-proton collisions at $\sqrt{s}=13 \mathrm{TeV}$, JHEP 01 (2018) 054 [arXiv: 1708.04188] [INSPIRE].

[22] ATLAS collaboration, Search for Higgs boson pair production in the $\gamma \gamma W W^{*}$ channel using pp collision data recorded at $\sqrt{s}=13 \mathrm{TeV}$ with the ATLAS detector, Eur. Phys. J. C 78 (2018) 1007 [arXiv: 1807.08567] [INSPIRE].

[23] ATLAS collaboration, Search for Higgs boson pair production in the $W W^{(*)} W W^{(*)}$ decay channel using ATLAS data recorded at $\sqrt{s}=13 \mathrm{TeV}$, JHEP 05 (2019) 124 [arXiv: 1811.11028] [INSPIRE].

[24] CMS collaboration, Combination of searches for Higgs boson pair production in proton-proton collisions at $\sqrt{s}=13 \mathrm{TeV}$, Phys. Rev. Lett. 122 (2019) 121803 [arXiv: 1811.09689] [INSPIRE].

[25] ATLAS collaboration, Combination of searches for Higgs boson pairs in pp collisions at $\sqrt{s}=13 \mathrm{TeV}$ with the ATLAS detector, Phys. Lett. B 800 (2020) 135103 [arXiv: 1906.02025] [INSPIRE].

[26] M.J. Dolan, C. Englert, N. Greiner and M. Spannowsky, Further on up the road: hhjj production at the LHC, Phys. Rev. Lett. 112 (2014) 101802 [arXiv:1310.1084] [INSPIRE]. 
[27] L.-S. Ling, R.-Y. Zhang, W.-G. Ma, L. Guo, W.-H. Li and X.-Z. Li, NNLO QCD corrections to Higgs pair production via vector boson fusion at hadron colliders, Phys. Rev. D 89 (2014) 073001 [arXiv: 1401.7754 ] [INSPIRE].

[28] M.J. Dolan, C. Englert, N. Greiner, K. Nordstrom and M. Spannowsky, hhjj production at the LHC, Eur. Phys. J. C $\mathbf{7 5}$ (2015) 387 [arXiv:1506.08008] [InSPIRE].

[29] F. Bishara, R. Contino and J. Rojo, Higgs pair production in vector-boson fusion at the LHC and beyond, Eur. Phys. J. C 77 (2017) 481 [arXiv:1611.03860] [InSPIRE].

[30] E. Arganda, C. Garcia-Garcia and M.J. Herrero, Probing the Higgs self-coupling through double Higgs production in vector boson scattering at the LHC, Nucl. Phys. B 945 (2019) 114687 [arXiv: 1807.09736] [INSPIRE].

[31] J. Baglio, A. Djouadi, R. Gröber, M.M. Mühlleitner, J. Quevillon and M. Spira, The measurement of the Higgs self-coupling at the LHC: theoretical status, JHEP 04 (2013) 151 [arXiv: 1212.5581] [INSPIRE].

[32] R. Frederix et al., Higgs pair production at the LHC with NLO and parton-shower effects, Phys. Lett. B 732 (2014) 142 [arXiv:1401.7340] [InSPIRE].

[33] F.A. Dreyer and A. Karlberg, Vector-Boson Fusion Higgs Pair Production at $N^{3} L O$, Phys. Rev. D 98 (2018) 114016 [arXiv: 1811.07906] [INSPIRE].

[34] F.A. Dreyer and A. Karlberg, Fully differential Vector-Boson Fusion Higgs Pair Production at Next-to-Next-to-Leading Order, Phys. Rev. D 99 (2019) 074028 [arXiv:1811.07918] [INSPIRE].

[35] F.A. Dreyer, A. Karlberg and L. Tancredi, On the impact of non-factorisable corrections in VBF single and double Higgs production, JHEP 10 (2020) 131 [arXiv:2005.11334] [INSPIRE].

[36] F.A. Dreyer, A. Karlberg, J.-N. Lang and M. Pellen, Precise predictions for double-Higgs production via vector-boson fusion, Eur. Phys. J. C 80 (2020) 1037 [arXiv:2005.13341] [INSPIRE].

[37] C. Englert, Q. Li, M. Spannowsky, M. Wang and L. Wang, VBS $W^{ \pm} W^{ \pm} H$ production at the HL-LHC and a $100 \mathrm{TeV}$ pp-collider, Int. J. Mod. Phys. A 32 (2017) 1750106 [arXiv: 1702.01930] [INSPIRE].

[38] K. Nordström and A. Papaefstathiou, VHH production at the High-Luminosity LHC, Eur. Phys. J. Plus 134 (2019) 288 [arXiv:1807.01571] [inSPIRE].

[39] T. Plehn, M. Spira and P.M. Zerwas, Pair production of neutral Higgs particles in gluon-gluon collisions, Nucl. Phys. B 479 (1996) 46 [Erratum ibid. 531 (1998) 655] [hep-ph/9603205] [INSPIRE].

[40] U. Baur, T. Plehn and D.L. Rainwater, Measuring the Higgs Boson Self Coupling at the LHC and Finite Top Mass Matrix Elements, Phys. Rev. Lett. 89 (2002) 151801 [hep-ph/0206024] [INSPIRE].

[41] Q. Li, Q.-S. Yan and X. Zhao, Higgs Pair Production: Improved Description by Matrix Element Matching, Phys. Rev. D 89 (2014) 033015 [arXiv:1312.3830] [INSPIRE].

[42] Q.-H. Cao, B. Yan, D.-M. Zhang and H. Zhang, Resolving the Degeneracy in Single Higgs Production with Higgs Pair Production, Phys. Lett. B 752 (2016) 285 [arXiv:1508.06512] [INSPIRE]. 
[43] Q.-H. Cao, G. Li, B. Yan, D.-M. Zhang and H. Zhang, Double Higgs production at the $14 \mathrm{TeV}$ LHC and a $100 \mathrm{TeV}$ pp collider, Phys. Rev. D 96 (2017) 095031 [arXiv:1611.09336] [INSPIRE].

[44] U. Baur, T. Plehn and D.L. Rainwater, Determining the Higgs Boson Selfcoupling at Hadron Colliders, Phys. Rev. D 67 (2003) 033003 [hep-ph/0211224] [INSPIRE].

[45] J. Ren, R.-Q. Xiao, M. Zhou, Y. Fang, H.-J. He and W. Yao, LHC Search of New Higgs Boson via Resonant Di-Higgs Production with Decays into 4W, JHEP 06 (2018) 090 [arXiv: 1706. 05980] [INSPIRE].

[46] U. Baur, T. Plehn and D.L. Rainwater, Probing the Higgs selfcoupling at hadron colliders using rare decays, Phys. Rev. D 69 (2004) 053004 [hep-ph/0310056] [INSPIRE].

[47] W. Yao, Studies of measuring Higgs self-coupling with $H H \rightarrow b \bar{b} \gamma \gamma$ at the future hadron colliders, in proceedings of the Community Summer Study 2013: Snowmass on the Mississippi (CSS2013), Minneapolis, MN, U.S.A., 29 July-6 August 2013, arXiv:1308.6302 [INSPIRE].

[48] F. Kling, T. Plehn and P. Schichtel, Maximizing the significance in Higgs boson pair analyses, Phys. Rev. D 95 (2017) 035026 [arXiv:1607.07441] [InSPIRE].

[49] J. Chang, K. Cheung, J.S. Lee, C.-T. Lu and J. Park, Higgs-boson-pair production $H(\rightarrow b \bar{b}) H(\rightarrow \gamma \gamma)$ from gluon fusion at the HL-LHC and HL-100 TeV hadron collider, Phys. Rev. D 100 (2019) 096001 [arXiv: 1804.07130] [INSPIRE].

[50] J.H. Kim, Y. Sakaki and M. Son, Combined analysis of double Higgs production via gluon fusion at the HL-LHC in the effective field theory approach, Phys. Rev. D 98 (2018) 015016 [arXiv: 1801.06093] [INSPIRE].

[51] H.-J. He, J. Ren and W. Yao, Probing new physics of cubic Higgs boson interaction via Higgs pair production at hadron colliders, Phys. Rev. D 93 (2016) 015003 [arXiv:1506.03302] [INSPIRE].

[52] A. Papaefstathiou, L.L. Yang and J. Zurita, Higgs boson pair production at the LHC in the $b \bar{b} W^{+} W^{-}$channel, Phys. Rev. D 87 (2013) 011301 [arXiv:1209.1489] [INSPIRE].

[53] U. Baur, T. Plehn and D.L. Rainwater, Examining the Higgs boson potential at lepton and hadron colliders: A Comparative analysis, Phys. Rev. D 68 (2003) 033001 [hep-ph/0304015] [INSPIRE].

[54] M.J. Dolan, C. Englert and M. Spannowsky, Higgs self-coupling measurements at the LHC, JHEP 10 (2012) 112 [arXiv:1206.5001] [INSPIRE].

[55] A.J. Barr, M.J. Dolan, C. Englert and M. Spannowsky, Di-Higgs final states augMT2ed selecting hh events at the high luminosity LHC, Phys. Lett. B 728 (2014) 308 [arXiv: 1309.6318] [INSPIRE].

[56] D.E. Ferreira de Lima, A. Papaefstathiou and M. Spannowsky, Standard model Higgs boson pair production in the $(b \bar{b})(b \bar{b})$ final state, JHEP 08 (2014) 030 [arXiv:1404.7139] [INSPIRE].

[57] J.K. Behr, D. Bortoletto, J.A. Frost, N.P. Hartland, C. Issever and J. Rojo, Boosting Higgs

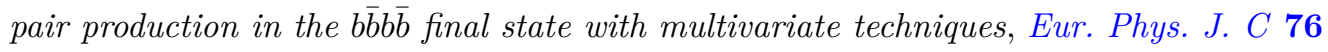
(2016) 386 [arXiv: 1512.08928] [INSPIRE].

[58] V. Barger, L.L. Everett, C.B. Jackson and G. Shaughnessy, Higgs-Pair Production and Measurement of the Triscalar Coupling at LHC (8,14), Phys. Lett. B 728 (2014) 433 [arXiv:1311.2931] [INSPIRE]. 
[59] A.J. Barr, M.J. Dolan, C. Englert, D.E. Ferreira de Lima and M. Spannowsky, Higgs Self-Coupling Measurements at a $100 \mathrm{TeV}$ Hadron Collider, JHEP 02 (2015) 016 [arXiv: 1412.7154] [INSPIRE].

[60] A. Papaefstathiou, Discovering Higgs boson pair production through rare final states at a $100 \mathrm{TeV}$ collider, Phys. Rev. D 91 (2015) 113016 [arXiv:1504.04621] [InSPIRE].

[61] Q. Li, Z. Li, Q.-S. Yan and X. Zhao, Probe Higgs boson pair production via the $3 \ell 2 j+\not t$ mode, Phys. Rev. D 92 (2015) 014015 [arXiv: 1503.07611] [INSPIRE].

[62] X. Zhao, Q. Li, Z. Li and Q.-S. Yan, Discovery potential of Higgs boson pair production through $4 \ell+\mathbb{E}$ final states at a $100 \mathrm{TeV}$ collider, Chin. Phys. C 41 (2017) 023105 [arXiv: 1604.04329] [INSPIRE].

[63] R. Contino et al., Physics at a $100 \mathrm{TeV}$ pp collider: Higgs and EW symmetry breaking studies, in CERN Yellow Reports 3, CERN, Geneva Switzerland (2017), pp. 255-440 [arXiv: 1606.09408] [INSPIRE].

[64] D. Gonçalves, T. Han, F. Kling, T. Plehn and M. Takeuchi, Higgs boson pair production at future hadron colliders: From kinematics to dynamics, Phys. Rev. D 97 (2018) 113004 [arXiv: 1802.04319] [INSPIRE].

[65] W. Kilian, T. Ohl and J. Reuter, WHIZARD: Simulating Multi-Particle Processes at LHC and ILC, Eur. Phys. J. C 71 (2011) 1742 [arXiv:0708.4233] [INSPIRE].

[66] J. Pumplin, D.R. Stump, J. Huston, H.L. Lai, P.M. Nadolsky and W.K. Tung, New generation of parton distributions with uncertainties from global QCD analysis, JHEP $\mathbf{0 7}$ (2002) 012 [hep-ph/0201195] [INSPIRE].

[67] J. Alwall et al., The automated computation of tree-level and next-to-leading order differential cross sections, and their matching to parton shower simulations, JHEP 07 (2014) 079 [arXiv: 1405.0301] [INSPIRE].

[68] M.L. Mangano, M. Moretti, F. Piccinini, R. Pittau and A.D. Polosa, ALPGEN, a generator for hard multiparton processes in hadronic collisions, JHEP 07 (2003) 001 [hep-ph/0206293] [INSPIRE].

[69] T. Sjöstrand, S. Mrenna and P.Z. Skands, A Brief Introduction to PYTHIA 8.1, Comput. Phys. Commun. 178 (2008) 852 [arXiv:0710.3820] [INSPIRE].

[70] M. Cacciari, G.P. Salam and G. Soyez, FastJet User Manual, Eur. Phys. J. C 72 (2012) 1896 [arXiv: 1111.6097] [INSPIRE].

[71] M. Cacciari, G.P. Salam and G. Soyez, The anti- $k_{t}$ jet clustering algorithm, JHEP 04 (2008) 063 [arXiv: 0802.1189] [INSPIRE].

[72] J.M. Butterworth, A.R. Davison, M. Rubin and G.P. Salam, Jet substructure as a new Higgs search channel at the LHC, Phys. Rev. Lett. 100 (2008) 242001 [arXiv:0802.2470] [INSPIRE].

[73] J. Thaler and K. Van Tilburg, Identifying Boosted Objects with N-subjettiness, JHEP 03 (2011) 015 [arXiv:1011.2268] [INSPIRE].

[74] S. Marzani, G. Soyez and M. Spannowsky, Looking inside jets: an introduction to jet substructure and boosted-object phenomenology, in Lecture Notes in Physics 958, Springer (2019) [arXiv:1901.10342] [INSPIRE]. 
[75] Y.L. Dokshitzer, G.D. Leder, S. Moretti and B.R. Webber, Better jet clustering algorithms, JHEP 08 (1997) 001 [hep-ph/9707323] [INSPIRE].

[76] M. Wobisch and T. Wengler, Hadronization corrections to jet cross-sections in deep inelastic scattering, in proceedings of the Workshop on Monte Carlo Generators for HERA Physics (Plenary Starting Meeting), 27-30 April 1998, Hamburg, Germany, pp. 270-279 [hep-ph/9907280] [INSPIRE].

[77] B.H. Denby, Neural Networks and Cellular Automata in Experimental High-energy Physics, Comput. Phys. Commun. 49 (1988) 429 [InSPIRE].

[78] Y. Freund and R.E. Schapire, A decision-theoretic generalization of on-line learning and an application to boosting, J. Comput. Syst. Sci. 55 (1997) 119 [INSPIRE].

[79] A. Hocker et al., TMVA - Toolkit for Multivariate Data Analysis, physics/0703039 [INSPIRE].

[80] G.F. Giudice, C. Grojean, A. Pomarol and R. Rattazzi, The Strongly-Interacting Light Higgs, JHEP 06 (2007) 045 [hep-ph/0703164] [INSPIRE].

[81] W. Kilian, S. Sun, Q.-S. Yan, X. Zhao and Z. Zhao, New Physics in multi-Higgs boson final states, JHEP 06 (2017) 145 [arXiv: 1702.03554] [INSPIRE].

[82] S. Das Bakshi, J. Chakrabortty, C. Englert, M. Spannowsky and P. Stylianou, CP violation at ATLAS in effective field theory, Phys. Rev. D 103 (2021) 055008 [arXiv:2009.13394] [INSPIRE].

[83] J. Ellis, C.W. Murphy, V. Sanz and T. You, Updated Global SMEFT Fit to Higgs, Diboson and Electroweak Data, JHEP 06 (2018) 146 [arXiv:1803.03252] [INSPIRE].

[84] M. Cacciari, G.P. Salam and G. Soyez, SoftKiller, a particle-level pileup removal method, Eur. Phys. J. C 75 (2015) 59 [arXiv: 1407.0408] [InSPIRE].

[85] F. Maltoni, K. Paul, T. Stelzer and S. Willenbrock, Color Flow Decomposition of QCD Amplitudes, Phys. Rev. D 67 (2003) 014026 [hep-ph/0209271] [INSPIRE].

[86] J. Gallicchio and M.D. Schwartz, Seeing in Color: Jet Superstructure, Phys. Rev. Lett. 105 (2010) 022001 [arXiv: 1001 . 5027] [INSPIRE].

[87] J.H. Kim, M. Kim, K. Kong, K.T. Matchev and M. Park, Portraying Double Higgs at the Large Hadron Collider, JHEP 09 (2019) 047 [arXiv: 1904.08549] [INSPIRE]. 